\title{
Complete post-separation of overlapping ultrasonic signals by combining hard and soft modeling
}

\author{
J. Martinsson $^{\mathrm{a}, *}$, F. Hägglund ${ }^{\mathrm{a}}$, J.E. Carlson ${ }^{\mathrm{b}}$ \\ ${ }^{a}$ EISLAB, Department of Computer Science and Electrical Engineering, Lulea University of Technology, SE-971 87 Luleå, Sweden \\ ${ }^{\mathrm{b}}$ Division of Systems and Interaction, Department of Computer Science and Electrical Engineering, Luleà University of Technology, SE-971 87 Luleå, Sweden
}

Received 22 October 2007; received in revised form 29 February 2008; accepted 6 March 2008

Available online 15 March 2008

\begin{abstract}
In some ultrasonic measurement situations, an adequate signal separation is difficult to achieve. A typical situation is material characterization of thin media using pulse-echo or through-transmission techniques, when the time-of-flight in the media is shorter than the emitted signal's time support. Separated signals are necessary to obtain accurate estimates of material properties and transit times. In this paper a new method is proposed that enables complete post-separation of measured coinciding signals. The method is based on a combination of hard physical and soft empirical models, which allows for a description of both known and unknown properties making a complete separation possible. The validity and limitations of the model and the separation results are thoroughly addressed. The proposed technique is verified using real measurements on thin dispersive samples and validated using residual analysis. The experimental results show a complete separation with uncorrelated and normally distributed residuals. The method enables characterization and/or flow analysis in difficult overlapping situations.
\end{abstract}

(C) 2008 Elsevier B.V. All rights reserved.

PACS: 43.60.+d; 43.35.Zc; 43.20.El; 43.35.Ns

Keywords: Separation; Overlapping; Coinciding; Superimposed; Thin layers; Ultrasonic; Echoes; Signals; Characterization

\section{Introduction}

When designing ultrasonic measurement systems based on pulse-echo or through-transmission techniques, adequate signal separation is often a requirement for the analysis step. In material characterization, separable signals are necessary to obtain accurate estimates of transit times and material properties. However, there are a number of factors which can prevent a complete separation. If the propagation distance in the medium is short (due to fixed dimensions, or deliberately kept short for highly attenuative materials), or if the signal's time support is long (due to low-frequency transducers or if high-energy and low-

\footnotetext{
* Corresponding author. Tel.: +46 920493138; fax: +46 920492191.

E-mail address: Jesper.Martinsson@1tu.se (J. Martinsson).
}

amplitude excitations are required), a complete separation can be difficult to achieve.

The problem can be divided into two categories: when there is no knowledge of the measured structure (e.g. the number of layers are unknown) and when there is knowledge of the structure.

For the case when there is no knowledge of the structure, a promising approach is to parameterize the signal waveforms (for a known number of waveforms) using a signal model of the ultrasonic echo $[1,2]$. However, to find an appropriate signal model one must have an accurate a priori information of the shape of the emitted ultrasonic signal and how it is affected by the media and the surrounding structure. A difficult and crucial step in using this approach is to find a valid signal model, that can capture the observed waveforms entirely and leave white residuals (uncorrelated errors). If the signal model is not general 
enough and the residuals contain any signal components which the model cannot reproduce, a complete separation cannot be achieved. A chosen signal model is often restricted to a certain type of signal waveform. This means that a large collection of signal models must be available to cope with different waveforms and measurement situations.

The second category is when information of the measured structure is available, for example, a material sample consisting of several layers. The required information is often limited to knowledge of the number layers within the structure, and the possibility to measure a reference signal (using a buffer rod or from a reference experiment). Several different approaches, based on both parametric and non-parametric techniques, have been proposed in the literature, [3-7], to cope with the overlapping problem for known structures. However, the main objective has been to retrieve estimates of a few physical parameters (thickness, phase velocity, density, attenuation) related to the investigated structure, rather than to capture all the systematic variations observed in the data. The result is that neglected effects, such as frequency dependent attenuation, dispersion, diffraction and alignment errors, are un-captured by the models, and the residuals contain variation originating from the neglected effects.

A necessary condition for a model to be able to accurately separate superimposed signals, is that all the observed dynamics can be described. This is, in general, also a necessary condition in order to obtain unbiased estimates of physical parameters from the observed data.

To separate the signals we must find a model that can describe the known structure and is general enough to capture both known and unknown effects. In this paper we use a combination of hard and soft modeling to solve this problem. ${ }^{1}$ A hard model structure is applied to describe multiple reflections and overlaps related to the specific measurement setup. A soft model structure is used to capture unknown dispersion and attenuation effects from the layers, and to handle unwanted diffraction and misalignment effects from the measurement setup.

The proposed method achieves complete signal separation in dispersive experimental situations (with no visible leftovers from the emitted signal). The method avails for characterization and flow analysis in overlapping situations. This paper is an extension of previous work, presented in [8].

The remaining part of the paper is organized as follows: In Section 2, the separation model is derived, the idea of combining hard and soft models is presented, and the soft model parameterization used in this study is stated. In Section 3 , aspects concerning parameter estimation, model validation, and stability constraints are addressed. Section 4 presents the key equations to separate overlapping signals,

\footnotetext{
${ }^{1}$ Open source MATLAB $\AA$ files, containing the derived separation method that produces the experimental results presented in this paper, is available at: www.sm.luth.se/ jesper/matlab/separation/example.zip (or upon request).
}

and it is shown how acoustic properties connected to layers within the measurement structure can be retrieved, followed by a thorough explanation of the validity of the separated signals. Section 5 contains a description of the measurement setup and the equipments used in the experimental study. In Section 6, estimation, model validation, and separation results are presented for two different experiments: long dispersive reverberation, and short dispersive reverberation. Section 7, contains a discussion addressing: sign invariance of parameters, characterization of layer properties, model extensions, soft model parameterizations, colored measurement noise, and how to deal with truncated measurements. Finally, conclusions can be found in Section 8, followed by an Appendix.

\section{Separation model}

This section begins with a derivation of the hard model structure for the specific measurement setup shown in Fig. 1, (designed for normal incidence pulse-echo measurements of thin samples). The hard structure describes the ideal case when attenuation, dispersion, diffraction, and misalignment effects are neglected and the structure contains only pure delays, reflection and transmission coefficients. This structure describes how the signals overlap and is essential to obtain separation. The hard structure is then expanded with soft structures to cope with a more realistic case when the above effects are included. Finally, details concerning parameterization of the soft models are presented.

Assuming that linear acoustic applies, there exist a linear system $\tilde{h}(\omega, \boldsymbol{\theta})$ that describes the relationship between the reference signal $\tilde{u}_{3}(\omega)$ and the response signal $\tilde{y}(\omega)$ as

$\tilde{y}(\omega)=\tilde{h}(\omega, \boldsymbol{\theta}) \tilde{u}_{3}(\omega)$.

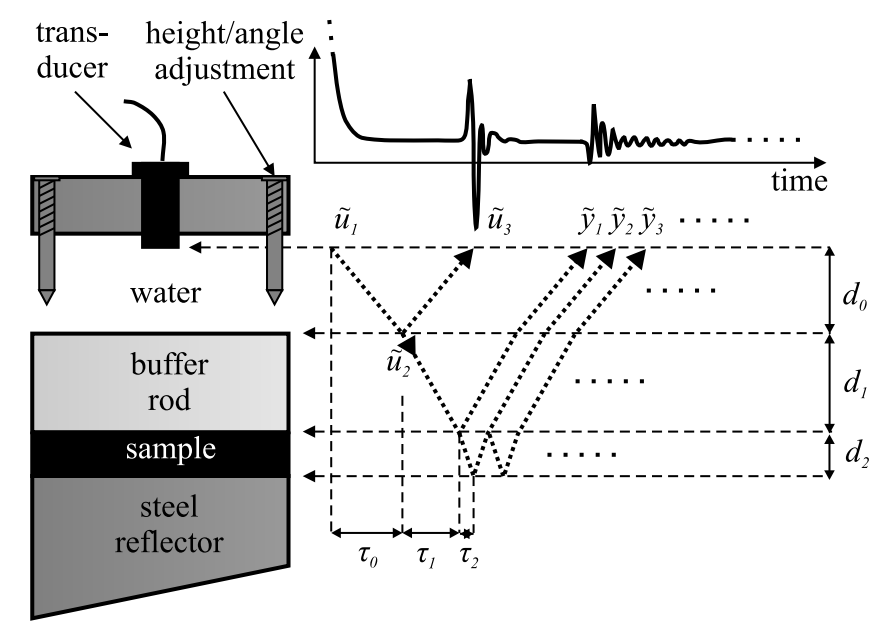

Fig. 1. Schematic figure over the measurement setup and the pulse-echo principle. The transducer emits an unknown sound wave $\tilde{u}_{1}(\omega)$. The reflection from the buffer $\operatorname{rod} \tilde{u}_{3}(\omega)$ and the multiple reflections from the sample space $\sum_{p=1}^{\infty} \tilde{y}_{p}(\omega)$ are then recorded. For this particular experiment four different mediums has been used: $\widetilde{M}_{0}(\omega)$ : water region, $\widetilde{M}_{1}(\omega)$ : plexiglas buffer, $\widetilde{M}_{2}(\omega)$ : sample, and $\widetilde{M}_{3}(\omega)$ : steel reflector. 
The reference signal is defined as the received reflection $\tilde{u}_{3}(\omega)$ from the water-buffer boundary, see Fig. 1. The response signal is defined as the summation of all the measured contributions obtained from the sample space

$\tilde{y}(\omega)=\sum_{p=1}^{\infty} \tilde{y}_{p}(\omega)$.

Here $\sim$ denotes Fourier transformed quantities and $\omega$ represents the angular frequency. In (1), $\boldsymbol{\theta}$ represents a vector containing the model parameters.

\subsection{Hard model}

Assume further that there are no attenuation and dispersion effects present, and that the measurement setup is perfectly aligned. Then the hard structure for $\tilde{h}(\omega, \boldsymbol{\theta})$ in (1) can be found by deriving the expressions for the measured contributions $\tilde{y}_{p}(\omega)$ from the sample space, using reflection and transmission coefficients related to each layer. The first two contributions

$\tilde{y}_{1}(\omega)=R_{12} T_{10} \mathrm{e}^{-\mathrm{j} \omega\left(2 \tau_{1}+\tau_{0}\right)} \tilde{u}_{2}(\omega)$,

$\tilde{y}_{2}(\omega)=T_{12} R_{23} T_{21} T_{10} \mathrm{e}^{-\mathrm{j} \omega\left(2\left(\tau_{1}+\tau_{2}\right)+\tau_{0}\right)} \tilde{u}_{2}(\omega)$,

are unique but the sequential contributions can be expressed in a recursive manner as

$\tilde{y}_{p}(\omega)=R_{21} R_{23} \mathrm{e}^{-\mathrm{j} \omega 2 \tau_{2}} \tilde{y}_{p-1}(\omega), \quad$ for $p \geqslant 3$.

Here $R_{l m}$ and $T_{l m}$ denote the (pressure) reflection and transmission coefficients, respectively, between the $l$ th and the $m$ th medium. The phase delay (time-of-flight) for the wave through the $m$ th material is represented by $\tau_{m}$ and $\tilde{u}_{2}(\omega)$ is defined as the pulse just inside the buffer rod, see Fig. 1.

The response signal, $\tilde{y}(\omega)$, can now be simplified as

$$
\begin{aligned}
\tilde{y}(\omega) & =\sum_{p=1}^{\infty} \tilde{y}_{p}(\omega)=\tilde{y}_{1}(\omega)+\sum_{p=2}^{\infty} \tilde{y}_{p}(\omega) \\
& =\tilde{y}_{1}(\omega)+\sum_{p=2}^{\infty}\left(R_{21} R_{23} \mathrm{e}^{-\mathrm{j} \omega 2 \tau_{2}}\right)^{p-2} \tilde{y}_{2}(\omega) \\
& =\tilde{y}_{1}(\omega)+\frac{1}{1-R_{21} R_{23} \mathrm{e}^{-\mathrm{j} \omega 2 \tau_{2}}} \tilde{y}_{2}(\omega),
\end{aligned}
$$

where the relationship

$\sum_{p=0}^{\infty} x^{p}=\frac{1}{1-x}, \quad$ for $|x|<1$

has been used in the last equality.

The last step in completing the path between the response signal $\tilde{y}(\omega)$ and the reference signal $\tilde{u}_{3}(\omega)$, is to find the relationship between $\tilde{u}_{2}(\omega)$ and $\tilde{u}_{3}(\omega)$. Using their mutual connection with the emitted pulse $\tilde{u}_{1}(\omega)$

$\tilde{u}_{2}(\omega)=T_{01} \mathrm{e}^{-\mathrm{j} \omega \tau_{0}} \tilde{u}_{1}(\omega)$,

$\tilde{u}_{3}(\omega)=R_{01} \mathrm{e}^{-\mathrm{j} \omega 2 \tau_{0}} \tilde{u}_{1}(\omega)$,

the relationship can be expressed as
$\tilde{u}_{2}(\omega)=\frac{T_{01}}{R_{01}} \mathrm{e}^{\mathrm{j} \omega \tau_{0}} \tilde{u}_{3}(\omega)$.

Using the relationship in (10), we can express (3) and (4) as

$$
\begin{aligned}
& \tilde{y}_{1}(\omega)=\frac{R_{12} T_{10} T_{01}}{R_{01}} \mathrm{e}^{-\mathrm{j} \omega 2 \tau_{1}} \tilde{u}_{3}(\omega), \\
& \tilde{y}_{2}(\omega)=\frac{T_{12} R_{23} T_{21} T_{10} T_{01}}{R_{01}} \mathrm{e}^{-\mathrm{j} \omega 2\left(\tau_{1}+\tau_{2}\right)} \tilde{u}_{3}(\omega) .
\end{aligned}
$$

Inserting the expressions (11) and (12) in (6), the hard model structure in (1) can be expressed as

$$
\begin{aligned}
\tilde{h}(\omega, \boldsymbol{\theta}) & =\left(R_{12}+\frac{T_{12} R_{23} T_{21} \mathrm{e}^{-\mathrm{j} \omega 2 \tau_{2}}}{1-R_{21} R_{23} \mathrm{e}^{-\mathrm{j} \omega 2 \tau_{2}}}\right) \frac{T_{10} T_{01}}{R_{01}} \mathrm{e}^{-\mathrm{j} \omega 2 \tau_{1}} \\
& =\left(R_{12}+\frac{\left(1-R_{12}^{2}\right) B \mathrm{e}^{-\mathrm{j} \omega 2 \tau_{2}}}{1+R_{12} B \mathrm{e}^{-\mathrm{j} \omega 2 \tau_{2}}}\right) A \mathrm{e}^{-\mathrm{j} \omega 2 \tau_{1}}
\end{aligned}
$$

where $A=\left(1-R_{01}^{2}\right) / R_{01}, B=R_{23}$ and $\boldsymbol{\theta}=\left[A, R_{12}, B, \tau_{1}, \tau_{2}\right]^{\mathrm{T}}$. The last equality is obtained using the relationships, $R_{l m}=-R_{m l}$ and $T_{l m}=1+R_{l m}$, between the transmission and reflection coefficients. The meaningless substitution $B=R_{23}$ is done to emphasis the connection with the combined model structure derived in Section 2.2.

\subsection{Combining hard and soft modeling}

In cases of diffraction, alignment errors, and frequency dependent attenuation and dispersion, the model structure in (13) is inadequate. However, if linear acoustic applies, the above effects can be described using linear systems and (1) is still valid. Assuming that the wave propagation through the $m$ th medium can be described by two linear systems:

- $\widetilde{M}_{m}(\omega)$ describing the attenuation and dispersion effects in the medium.

- $\widetilde{D}\left(\omega, \tilde{\xi}_{m}(\omega)\right)$ describing diffraction and alignment effects that depends on the total number of propagated wave numbers $\tilde{\xi}_{m}(\omega)=d_{m} \tilde{k}_{m}(\omega)$, where $d_{m}$ and $\tilde{k}_{m}(\omega)$ are the thickness and frequency dependent wave number, respectively.

Note that in the following analysis the $\omega$ dependency related to all the $\sim$ quantities has been omitted for notational simplicity. The derivation steps follow analogously to those in Section 2.1, with the addition of the linear models $\widetilde{M}_{m}$ and $\widetilde{D}\left(\widetilde{\xi}_{m}\right)$.

Introducing the soft model structures $\widetilde{M}_{m}$ and $\widetilde{D}\left(\tilde{\xi}_{m}\right)$, (3)-(5) are expanded to

$$
\begin{aligned}
\tilde{y}_{1}= & \widetilde{M}_{1}^{2} \widetilde{M}_{0} \widetilde{D}\left(2 \tilde{\xi}_{1}+\tilde{\xi}_{0}\right) \\
& \times \widetilde{R}_{12} \widetilde{T}_{10} \mathrm{e}^{-\mathrm{j} \omega\left(2 \tau_{1}+\tau_{0}\right)} \tilde{u}_{2}, \\
\tilde{y}_{2}= & \widetilde{M}_{1}^{2} \widetilde{M}_{2}^{2} \widetilde{M}_{0} \widetilde{D}\left(2 \tilde{\xi}_{1}+2 \tilde{\xi}_{2}+\tilde{\xi}_{0}\right) \\
& \times \widetilde{T}_{12} \widetilde{R}_{23} \widetilde{T}_{21} \widetilde{T}_{10} \mathrm{e}^{-\mathrm{j} \omega\left(2\left(\tau_{1}+\tau_{2}\right)+\tau_{0}\right)} \tilde{u}_{2},
\end{aligned}
$$




$$
\begin{aligned}
\tilde{y}_{p}= & \widetilde{M}_{2}^{2} \frac{\widetilde{D}\left(2 \tilde{\xi}_{1}+(p-1) 2 \tilde{\xi}_{2}+\tilde{\xi}_{0}\right)}{\widetilde{D}\left(2 \tilde{\xi}_{1}+(p-2) 2 \tilde{\xi}_{2}+\tilde{\xi}_{0}\right)} \\
& \times \widetilde{R}_{21} \widetilde{R}_{23} \mathrm{e}^{-\mathrm{j} \omega 2 \tau_{2}} \tilde{y}_{p-1}, \quad \text { for } p \geqslant 3 .
\end{aligned}
$$

In this case the phase velocity and the phase delay are frequency dependent, which means that the reflection and transmission coefficients are frequency dependent. Here $\tau_{m}$ should be considered as the overall frequency independent phase delay (or dead-time). Observe that the last terms in (14)-(16) are identical to those in (3)-(5) and corresponds to lossless wave propagation. The additional terms are associated with the previously neglected effects (attenuation, dispersion, diffraction, misalignment).

Using relatively high frequencies combined with a small sample space (small $\widetilde{\xi}_{2}$ ), the diffraction/alignment ratio in (16) can be considered as independent of $p$ and approximated as

$\frac{\widetilde{D}\left(2 \tilde{\xi}_{1}+(p-1) 2 \tilde{\xi}_{2}+\tilde{\xi}_{0}\right)}{\widetilde{D}\left(2 \tilde{\xi}_{1}+(p-2) 2 \tilde{\xi}_{2}+\tilde{\xi}_{0}\right)} \approx \frac{\widetilde{D}\left(2 \tilde{\xi}_{1}+2 \tilde{\xi}_{2}+\tilde{\xi}_{0}\right)}{\widetilde{D}\left(2 \tilde{\xi}_{1}+\tilde{\xi}_{0}\right)}=\widetilde{E}$.

The above approximation does not mean that diffraction and alignment effects are negligible for small sample spaces, even though they might be small, it means only that their ratio can be considered independent of $p$, i.e., the ratio between the diffraction affecting $\tilde{y}_{p}$ and $\tilde{y}_{p+1}$ is approximated as the same as the ratio between the diffraction affecting $\tilde{y}_{p+1}$ and $\tilde{y}_{p+2}$.

Using the approximation in (17), the response signal $\tilde{y}$ can be expressed, analogous to (6), as

$\tilde{y}=\tilde{y}_{1}+\frac{1}{1-\widetilde{R}_{21} \widetilde{R}_{23} \widetilde{M}_{2}^{2} \widetilde{E} \mathrm{e}^{-\mathrm{j} \omega 2 \tau_{2}}} \tilde{y}_{2}$.

Eqs. (8) and (9) are rewritten as

$\tilde{u}_{2}=\widetilde{M}_{0} \widetilde{D}\left(\tilde{\xi}_{0}\right) \widetilde{T}_{01} \mathrm{e}^{-\mathrm{j} \omega \tau_{0}} \tilde{u}_{1}$,

$\tilde{u}_{3}=\widetilde{M}_{0}^{2} \widetilde{D}\left(2 \tilde{\xi}_{0}\right) \widetilde{R}_{01} \mathrm{e}^{-\mathrm{j} \omega 2 \tau_{0}} \tilde{u}_{1}$,

resulting in changing the relationship in (10) between $\tilde{u}_{2}$ and $\tilde{u}_{3}$ to

$\tilde{u}_{2}=\frac{\widetilde{D}\left(\tilde{\xi}_{0}\right) \widetilde{T}_{01}}{\widetilde{M}_{0} \widetilde{D}\left(2 \tilde{\xi}_{0}\right) \widetilde{R}_{01}} \mathrm{e}^{\mathrm{j} \omega \tau_{0}} \tilde{u}_{3}$.

Inserting the expressions (14), (15), (21) in (18), the combined model structure in (1) can be expressed as

$\tilde{h}(\boldsymbol{\theta})=\left(\widetilde{R}_{12}+\frac{\left(1-\widetilde{R}_{12}^{2}\right) \widetilde{B} \mathrm{e}^{-\mathrm{j} \omega 2 \tau_{2}}}{1+\widetilde{R}_{12} \widetilde{B} \mathrm{e}^{-\mathrm{j} \omega 2 \tau_{2}}}\right) \widetilde{A} \mathrm{e}^{-\mathrm{j} \omega 2 \tau_{1}}$,

where

$\widetilde{A}=\widetilde{M}_{1}^{2} \frac{\widetilde{D}\left(2 \tilde{\xi}_{1}+\tilde{\xi}_{0}\right) \widetilde{D}\left(\tilde{\xi}_{0}\right)}{\widetilde{D}\left(2 \tilde{\xi}_{0}\right)} \frac{\left(1-\widetilde{R}_{01}^{2}\right)}{\widetilde{R}_{01}}$,

$\widetilde{B}=\widetilde{M}_{2}^{2} \widetilde{E} \widetilde{R}_{23}$

see Appendix A.1. Observe that the hard model in (13) is a special case of the combined model in (22), when attenuation, dispersion, diffraction and misalignment are neglected $\left(\widetilde{M}_{m}=\widetilde{D}\left(\widetilde{\xi}_{m}\right)=1\right.$, for all layers $\left.m\right)$.
If the sample space is small relative to the time support of the pulse (discussed in Section 1), then an overlapping waveform is measured ( $\tilde{y}_{p}$ is unknown), and information on how each layer is affecting each response is hidden. To separate the responses, $\tilde{y}_{p}$, the combined model in (22) must be applied, together with a suitable parameterization of $\widetilde{A}, \widetilde{R}_{12}$ and $\widetilde{B}$.

\subsection{Parameterization of the soft models}

Some of the effects described by the models $\widetilde{A}, \widetilde{R}_{12}$ and $\widetilde{B}$ have unknown physical structures, e.g., diffraction, misalignment, and acoustic properties of the investigated sample. This means that a general structure is needed to parameterize these models. In this paper a phase compensated finite impulse response (FIR) filter is used to parameterize $\widetilde{A}, \widetilde{R}_{12}$ and $\widetilde{B}$ as

$\widetilde{A}(\mathbf{a})=\mathrm{e}^{\mathrm{j} \omega T_{\mathrm{s}}\left(n_{a}-1\right) / 2} \sum_{p=1}^{n_{a}} a_{p} \mathrm{e}^{-\mathrm{j} \omega T_{\mathrm{s}}(p-1)}$,
$\widetilde{R}_{12}(\mathbf{r})=\mathrm{e}^{\mathrm{j} \omega T_{\mathrm{s}}\left(n_{r}-1\right) / 2} \sum_{p=1}^{n_{r}} r_{p} \mathrm{e}^{-\mathrm{j} \omega T_{\mathrm{s}}(p-1)}$,
$\widetilde{B}(\mathbf{b})=\mathrm{e}^{\mathrm{j} \omega T_{\mathrm{s}}\left(n_{b}-1\right) / 2} \sum_{p=1}^{n_{b}} b_{p} \mathrm{e}^{-\mathrm{j} \omega T_{\mathrm{s}}(p-1)}$,

where $n_{a}, n_{r}$ and $n_{b}$ are the dimensions of the parameter vectors $\mathbf{a}, \mathbf{r}$ and $\mathbf{b}$ respectively, and $T_{\mathrm{s}}$ denotes the sampling period. The term $\mathrm{e}^{\mathrm{j} \omega T_{\mathrm{s}}(n,-1) / 2}$ represents the phase compensation part and shifts the FIR so that the middle tap corresponds to zero delay (for odd $n$.).

The FIR filter describing the reflection coefficient $\widetilde{R}_{12}$ in (26) is restricted to a type I linear-phase FIR filter [9], i.e., the impulse response is symmetric $r_{p}=r_{n_{r}-p+1}$. This means that $\widetilde{R}_{12}$ is confined to be a real-valued frequency dependent quantity, i.e., no phase shifts other than $\pm \pi$ are associated with reflection and transmission. The linear-phase restriction in (26) can be relaxed, allowing for complex-valued reflection and transmission coefficients, e.g., in case of incomplete or partially contacting interfaces $[10,11]$.

Using a FIR representation of the soft models, the parameter vector in (22) is expanded to $\boldsymbol{\theta}=\left[\mathbf{a}^{\mathrm{T}}, \check{\mathbf{r}}^{\mathrm{T}}, \mathbf{b}^{\mathrm{T}}, \tau_{1}, \tau_{2}\right]^{\mathrm{T}}$, where $\check{\mathbf{r}}$ contains the first $\left(n_{r}+1\right) / 2$ elements of $\mathbf{r}$ due to the imposed linear-phase symmetry.

Note that the hard model structure in (13) is equivalent to the combined model structure in (22) using single tap FIR filters $\left(n_{a}=n_{b}=n_{r}=1\right)$.

\section{Parameter estimation}

This section begins with a short description of the parameter estimation techniques used to find the parameters to the separation model. Comments regarding stability constraints and finite reflections are addressed. Finally, issues concerning model selection and model validation are given. 
Given data from a pulse-echo experiment, the unknown model parameters are estimated from discrete Fourier transformed data [12], using a weighted nonlinear leastsquares fit (A.31) in Appendix A.3. The Levenberg-Marquardt optimization method [13] is used to maximize the likelihood function, expanding the search space and handling ill conditioned Hessians.

At the start of the optimization, all the FIR filter taps are set to zero except for the center tap. This initial set allows for an inverse description of non-minimum phase filters. To avoid local minima, the initial guess for the center taps are retrieved from optimized values obtained using the hard model structure in (13) (equivalent to (22) with single tap filters).

\subsection{Stability constraints and finite reflections}

Preserving the stability of $\tilde{h}(\boldsymbol{\theta})$ in (22) during the optimization process is an issue. The stability constraint is imposed by (7) and relates to the physical properties of the model. The problem is that (7) is valid only if $|x|<1$, and if this constraint is not included in the optimization, a better fit can in some cases be achieved for $|x|>1$ when (7) is invalid. The straightforward way to deal with this problem is to use a constrained optimization subjected to $\left|\widetilde{R}_{12} \widetilde{B}\right|<1 \forall \omega \leqslant \pi F_{\mathrm{s}}$. However, to avoid the increased complexity of a constrained optimization, the parameters can be estimated using a finite number of elements in (2). This approach can be motivated since only a finite number of reflections can be observed within the measurement window. This means that (2) and (7) is replaced with

$$
\begin{aligned}
& \tilde{y}(\omega)=\sum_{p=1}^{P_{0}} \tilde{y}_{p}(\omega), \\
& \sum_{p=0}^{P_{0}} x^{p}=\frac{1-x^{P_{0}}}{1-x}, \quad \text { for }|x| \neq 1,
\end{aligned}
$$

where $P_{0}$ is the number of observed elements within the measurement window. Using a finite number of elements a constrained optimization is avoided, since (29), in contrary to (7), increases rapidly if $|x|>1$ and $P_{0}$ is large. The $x^{P_{0}}$ part in (29) will act as a barrier function [13], enforcing the constraint.

For a finite number of observed elements, $P_{0}$, the model structures in (13) and (22) are replaced by

$$
\begin{aligned}
\tilde{h}(\boldsymbol{\theta}) & =\left(\widetilde{R}_{12}(\mathbf{r})+\frac{\left(1-\widetilde{R}_{12}^{2}(\mathbf{r})\right) \widetilde{B}(\mathbf{b}) \mathrm{e}^{-\mathrm{j} \omega 2 \tau_{2}}}{1+\widetilde{R}_{12}(\mathbf{r}) \widetilde{B}(\mathbf{b}) \mathrm{e}^{-\mathrm{j} \omega 2 \tau_{2}}}\right. \\
& \left.\times\left(1-\left(-\widetilde{R}_{12}(\mathbf{r}) \widetilde{B}(\mathbf{b}) \mathrm{e}^{-\mathrm{j} \omega 2 \tau_{2}}\right)^{P_{0}}\right)\right) \widetilde{A}(\mathbf{a}) \mathrm{e}^{-\mathrm{j} \omega 2 \tau_{1}},
\end{aligned}
$$

see Section 3.1. Note that if $P_{0} \rightarrow \infty$, then and (30) $\rightarrow$ (22) if $\left|\widetilde{R}_{12}(\mathbf{r}) \widetilde{B}(\mathbf{b})\right| \leqslant 1$, otherwise $|(30)| \rightarrow \infty$.

\subsection{Model selection and validation}

To prevent over-parameterization, the minimum description length (MDL) is used [14,15,12], to find the appropriate number of FIR parameters. The number of filter taps $n_{a}, n_{r}$ and $n_{b}$ are varied individually until the MDL is obtained.

To make sure that the model is capable of describing the true system we have to validate its performance. The key concept here is to examine the residuals (the part of the response $y(t)$ that the model could not reproduce) to determine the model's validity, Appendix A.5, [15]. If the residuals contain components other than noise, a complete separation cannot be claimed. For time-limited signals (pulses), a visual examination of the residuals for non-stationary variations can also detect inadequate modeling.

To preserve the linear-phase restriction on the reflection filter $\widetilde{R}_{12}$ in (26), and to always allow a center tap for the initial guess (Section 3), the number of taps are restricted to an odd number when searching for the MDL.

\section{Signal separation}

This section begins by stating the key equations, essential to separate the coinciding responses $\tilde{y}_{p}$. Then it is shown how acoustic properties connected to the layers can be retrieved from the soft models. Finally, a thoroughly explanation of the validity of the signal separation is presented and limitations are discussed.

Once the correct model order is found and validated, and an estimate $\hat{\boldsymbol{\theta}}=\left[\hat{\mathbf{a}}^{\mathrm{T}}, \hat{\mathbf{r}}^{\mathrm{T}}, \hat{\mathbf{b}}^{\mathrm{T}}, \hat{\tau}_{1}, \hat{\tau}_{2}\right]^{\mathrm{T}}$ of the parameter vector is obtained, the coinciding signals can be separated by returning to the basic equations in which the model were build upon.

The first separated signal can be retrieved by inserting (21) in (14), and using the relationship in (23) as

$\hat{\tilde{y}}_{1}=\widetilde{R}_{12}(\hat{\mathbf{r}}) \widetilde{A}(\hat{\mathbf{a}}) \mathrm{e}^{-\mathrm{j} \omega 2 \hat{\tau}_{1}} \tilde{u}_{3}^{\prime}$.

Here $\tilde{u}_{3}^{\prime}$ denotes the measured reference signal $\tilde{u}_{3}$ in Fig. 1, and denotes estimate. The sequential signals can be found using (31) together with

$\hat{\tilde{y}}_{2}=\frac{\left(1-\widetilde{R}_{12}^{2}(\hat{\mathbf{r}})\right)}{\widetilde{R}_{12}(\hat{\mathbf{r}})} \widetilde{B}(\hat{\mathbf{b}}) \mathrm{e}^{-\mathrm{j} \omega 2 \hat{\tau}_{2}} \hat{\tilde{y}}_{1}$,
$\hat{\tilde{y}}_{p}=-\widetilde{R}_{12}(\hat{\mathbf{r}}) \widetilde{B}(\hat{\mathbf{b}}) \mathrm{e}^{-\mathrm{j} \omega 2 \hat{\tau}_{2} \hat{\tilde{y}}_{p-1}, \quad \text { for } p>2,}$

simply derived by combining (14)-(17), and using the relationship in (24). Using (31)-(33) above, the overlapping signals can be separated.

\subsection{Estimating acoustic properties from soft models}

In addition to the separated waveforms, information of the acoustic properties of the layers can be retrieved from the estimated soft models, since they contain information of the medium $\left(\widetilde{M}_{m}\right.$ and $\left.\tau_{m}\right)$. However, the soft models in (23) and (24) also contain elements related to diffraction, 
misalignment and reflections, needed to be known (or negligible).

For example, the acoustic properties of the investigated sample can only be retrieved from the soft models if the diffraction and misalignment effects described by $\widetilde{E}$, and the reflection term $\widetilde{R}_{23}$ are known. If these conditions are met, then $\widetilde{M}_{2}^{2}=\widetilde{B} / \widetilde{E} \widetilde{R}_{23}$ from (24), and the attenuation and the phase velocity related to the sample can be estimated [16] as

$\hat{\tilde{\alpha}}(\omega)=\frac{-\ln \left(\left|\hat{\widetilde{M}}_{2}^{2}\right|\right)}{2 \hat{d}_{2}}=\frac{\ln (|\hat{\widetilde{E}}|)+\ln \left(\left|\hat{\widetilde{R}}_{23}\right|\right)-\ln (|\hat{\widetilde{B}}|)}{2 \hat{d}_{2}}$,

$\hat{\tilde{c}}_{p}(\omega)=\frac{-\omega 2 \hat{d}_{2}}{\angle \hat{\tilde{M}}_{2}^{2}-\omega 2 \hat{\tau}_{2}}=\frac{-\omega 2 \hat{d}_{2}}{\angle \hat{\tilde{B}}-\angle \hat{\tilde{E}}-\angle \hat{\tilde{R}}_{23}-\omega 2 \hat{\tau}_{2}}$,

respectively. In (34) and (35), $\hat{d}_{2}$ represents the estimated thickness of the sample, see Fig. 1.

\subsection{Validating the signal separation results}

A question that naturally arises is how to validate the signal separation. Investigating the residuals is one way to tell us that the applied model, at least, captures the total response. Another test is to take the sum of the separated signals, and see that it builds up the total response. However, it does not say so much about the validity and uniqueness of each separated signal obtained using (31)-(33). In other words we must answer the question: is there another set of separated signals that produce the same total response with white residuals?

For the ideal case, when the hard model structure applies, the answer is no, there exist only one unique separation solution that produces white residuals, under the condition that the reference and response signals are informative enough $[15,12,17]$.

As mentioned in Section 2.1, the design of the hard model structure is based on the specific measurement setup used and strictly depends on each layer within the measurement setup. The hard structure describes the setup under ideal conditions using a total amount of five parameters $\boldsymbol{\theta}=\left[A, R_{12}, B, \tau_{1}, \tau_{2}\right]^{\mathrm{T}}$. Remember that the structure is derived from infinite/finite reflections (6) using the geometric sum in (7)/(29), and can therefore be decoupled uniquely into each reflection using (3)-(5) as in Section 4.

If ideal conditions are met, and the reference and response signals are informative enough, there exist only two set of parameter values that will give the maximum likelihood solution and produce white residuals, $\hat{\boldsymbol{\theta}}=\left[\hat{A}, \hat{R}_{12}, \hat{B}, \hat{\tau}_{1}, \hat{\tau}_{2}\right]^{\mathrm{T}}$ and $\hat{\boldsymbol{\theta}}=\left[-\hat{A},-\hat{R}_{12},-\hat{B}, \hat{\tau}_{1}, \hat{\tau}_{2}\right]^{\mathrm{T}}$. Two solutions with the same likelihood are the result of the sign invariance discussed in Section 7.1, and the sign difference does not influence neither the separation nor the total response. If any of these two solutions are found, they will produce the same unique separation solution. A proof of this can be found by examining the eigenvalues of the Fisher information matrix (FIM), [12,17], of the hard model structure. For reasonable values of $\boldsymbol{\theta}$, i.e., $R_{01} \neq\{0,1\}$, $R_{12} \neq\{0,1\}$ and $\tau_{m} \neq 0$, and informative signals, the FIM has positive non-zero eigenvalues (full rank, positive definite matrix) and unique solutions can be found.

Note that the FIM F $(\hat{\boldsymbol{\theta}})$ is closely related to the Hessian of the cost-function in (A.34). If an eigenvalue of the Hessian is zero for a given estimate $\hat{\boldsymbol{\theta}}$, the cost-function is shaped like an half-pipe, and a unique solution cannot be found.

For the combined model structure case, the answer to uniqueness depends on the length of the FIR filters representing $\widetilde{A}, \widetilde{R}_{12}$ and $\widetilde{B}$. A way to investigate the dependency between the physical structure and the soft models, is to investigate the total impulse response of the combined model structure as the number of filter taps of the soft models increases. Decoupling the geometric sum in (30) gives

$$
\begin{aligned}
\tilde{h}(\boldsymbol{\theta}, \omega)= & \widetilde{R}_{12} \widetilde{A} \mathrm{e}^{-\mathrm{j} \omega 2 \tau_{1}}+\left(1-\widetilde{R}_{12}^{2}\right) \widetilde{B} \widetilde{A} \\
& \times \sum_{p=2}^{P_{0}}\left(-\widetilde{R}_{12} \widetilde{B}\right)^{p-2} \mathrm{e}^{-\mathrm{j} \omega 2\left(\tau_{1}+\tau_{2}(p-1)\right)} .
\end{aligned}
$$

The total impulse response of the model in (36) using single taps (hard model) is

$h(\boldsymbol{\theta}, t)=\beta_{1} \delta_{-2 \tau_{1}}+\beta_{2} \sum_{p=2}^{P_{0}} \beta_{3}^{p-2} \delta_{-2 \tau_{1}-2(p-1) \tau_{2}}$,

where

$\beta_{1}=R_{12} A$,

$\beta_{2}=\left(1-R_{12}^{2}\right) B A$,

$\beta_{3}=-R_{12} B$

and $\delta_{-\tau}=\delta(t-\tau)$ represents a Dirac impulse function located at time $\tau$. We know from the FIM, that using a model with single taps (the hard model) results in a unique separation. The impulse response in (37) produces a series of impulses, originating from the reflections within the thin layer. Notice that the relative distance between each impulse is $2 \tau_{2} / T_{\mathrm{s}}$ samples.

Let us examine the total impulse response of (36), using an arbitrary number of taps. The impulse response is given by

$h(\boldsymbol{\theta}, t)=\beta_{1} * \delta_{-2 \tau_{1}}+\beta_{2} * \sum_{p=2}^{P_{0}} \beta(p-2) * \delta_{-2 \tau_{1}-2(p-1) \tau_{2}}$,

where

$\beta_{1}=r_{12} * a$,

$\beta_{2}=\left(1-r_{12} * r_{12}\right) * b * a$,

$\beta(p-2)= \begin{cases}1, & p=2, \\ \beta_{3} * \beta_{3} * \cdots * \beta_{3},(p-2) \text { convs., } & p>2,\end{cases}$

$\beta_{3}=-r_{12} * b$,

and where $a, r_{12}$ and $b$ represents the finite impulse response of $\widetilde{A}, \widetilde{R}_{12}$ and $\widetilde{B}$, respectively, and $*$ denotes convo- 
lution. From (41), we can at least conclude that a unique solution (unique impulse response) is obtained if the length of the FIR in $\beta_{1}$ is smaller than $2 \tau_{2} / T_{\mathrm{s}}$ samples. If this condition is satisfied then the FIR $\beta_{1} * \delta_{-2 \tau_{1}}$, located at $2 \tau_{1}$, does not interfere with the next term $(p=2)$ in the geometric sum $\beta_{2} * \delta_{-2 \tau_{1}-2 \tau_{2}}$, located at $2 \tau_{1}+2 \tau_{2}$, and consequently the FIM has full set of positive non-zero eigenvalues.

However, for thin and dispersive layers (small $\tau_{2}$ and many taps), this condition is often violated. It does not mean that a non-unique situation will emerge. The term $\beta_{1} * \delta_{-2 \tau_{1}}$ will overlap with the next term $\beta_{2} * \delta_{-2 \tau_{1}-2 \tau_{2}}$, but the physical restriction on how $\beta_{1}$ and $\beta_{2}$ are constructed, is still going to prevent its building components $a, r_{12}$ and $b$ from varying in an arbitrary way.

The question is then: how much overlap between these two terms is tolerable before the physical restrictions break down? An examination of the eigenvalues of the FIM, calculated using similar signal condition and parameter values as in the two experiments conducted in this study, indicates that if $\tau_{2} / T_{\mathrm{s}} \geqslant\left(n_{a}+\left(n_{r}-1\right) / 2+1\right) / 4$ the FIM has full set of positive non-zero eigenvalues and a unique solution (unique impulse response) can be retrieved. A condition that is well met for both experiments in Section 6. If the linear-phase restriction on $\widetilde{R}_{12}$ is relaxed, see Section 2.3, the condition is replaced by $\tau_{2} / T_{\mathrm{s}} \geqslant\left(n_{a}+n_{r}\right) / 4$.

The problem of multiple solutions with white residuals, arises when the general soft models get too much freedom and captures dynamics that the structure is suppose to handle (the FIM is singular). For example, an extreme case would be if $n_{r}=n_{b}=1$, and $n_{a}=N$, where $N$ is the number of samples. Then the FIR filter represented by the soft model $A$ would capture everything, independent of the values of the other parameters. But this extreme case would certainly be prevented by the MDL criterion, due to the large amount of parameters (degrees of freedom). The MDL criterion prevents a solution where the soft models get too much freedom, since it searches for a solution with the minimum amount of parameters and takes maximum advantage of the dynamics generated by the physical structure incorporated into the combined model. If the soft models get too much freedom, the separated signals would look very strange, since a correct separation result depends on having a correct description of the physical structure.

\section{Experimental setup}

The experimental setup consists of a broadband piezoelectric transducer mounted on custom built measurement cell consisting of a water region $23.3 \pm 0.1 \mathrm{~mm}$ and a buffer rod made of $10.03 \pm 0.01 \mathrm{~mm}$ plexiglass. The sample space is located between the buffer rod and a stainless steel reflector, see Fig. 1. A pulser/receiver (5073PR, Panametrics, Waltham, MA, USA) was used to excite the transducer and amplify the received signal. The signal was then digitized using an oscilloscope card (CompuScope 12400, Gage
Applied Technologies Inc., Lachine, QC Canada) with 12bit resolution and using a sampling rate of $100 \mathrm{MHz}$. The measurements were made in a temperature controlled room, at $20 \pm 1{ }^{\circ} \mathrm{C}$.

The transducer is mounted in the middle of an aluminium disc. The disc rests on the buffer rod at three points, Fig. 1, in a tripod configuration. These points can be adjusted in height independently and dependently, to adjust the angle and height to the buffer (thickness of the water layer). Using this configuration, normal angles can be achieved and the water region thickness can be varied to obtain a separate reference echo $\left(\tilde{u}_{3}\right.$ separate from $\tilde{y})$.

Two different dispersive test samples were used in this study: high viscous marine transmission fluid [18], and glycerin. The pulse-echo tests were performed using a broadband piezoelectric transducer with center frequency of $5 \mathrm{MHz}$ (V3456, Panametrics, Waltham, MA, USA). To obtain an adequate SNR (and overlapping echoes), a small sample space was designed using a $0.15 \pm 0.02 \mathrm{~mm}$ washer between the buffer rod and the reflector. The washer is made of an elastic material to keep the fluid inside the sample space. The elasticity contributes to an estimated uncertainty $\pm 0.02 \mathrm{~mm}$ in sample distance.

\section{Results}

In this section the estimation, validation and separation results are presented for measurements in a high viscous marine transmission fluid and in glycerin. The objective is to show separability for two different overlapping waveforms: long dispersive reverberation, and short dispersive reverberation. The first fluid produce a long dispersive response (longer reverberation), and the other fluid on the contrary results in a very short dispersive reverberation (good impedance match to the buffer). These two situations complement each other by producing data with different separation and estimation difficulties. Both fluids are quite dispersive (high viscosity), hence, ideal for evaluating the combined structure when the hard structure assumptions are violated, see Sections 2.1 and 2.2.

In the optimization process $P_{0}=30$ reflections are used, see Section 3.1, which is approximately the amount spanned by the measurement window and well above the amount required to capture all reflections with significant amplitude. Due to the high sampling frequency (20 times the center frequency of the transducer) the parameter vector $\boldsymbol{\theta}$ are identified from information contained in the first quarter of the available frequency band, to reduce the time complexity of the estimation procedure. Note that it is only the estimate of the parameter vector $\boldsymbol{\theta}$ that are confined to this frequency region, and not the measured, estimated or shown signals and residuals. To remove DC components, trends and low-frequency disturbances, the measured signals are high-pass filtered using a discrete IIR filter, see Appendix A.2. 


\subsection{Long dispersive reverberation: separation and estimation results in marine transmission fluid (SAE90)}

Fig. 2 shows the measured reference signal $u_{3}^{\prime}[n]$ using the $5 \mathrm{MHz}$ transducer. Here ' denotes measured quantity, see Appendix A.3.

In Fig. 3, estimation results can be seen for the case where only the hard model structure, (30) with $n_{a}=n_{r}=n_{b}=1$, is used to estimate the response signal. A large systematic variation is visible in the residuals, Fig. 3a and $b$, indicating presence of un-modeled dynamics originating from diffraction, dispersion, attenuation and alignment effects neglected by the model, discussed in Section 2.1. This conclusion is supported by the highly autocorrelated residual sequence in Fig. 3c, where the tails $(k \neq 0)$ clearly violates the confidence region. In Fig. 3d, a normal probability plot can be observed for the residuals. From this plot a large $\mathrm{S}$-shaped deviation from the line can be seen, indicating a non-normal distribution. The Anderson-Darling test [19] for normality gives a test statistic of $A^{2}=103.47$ which is well above the $\alpha=0.01$ threshold of $A_{\text {critical }}^{2}=0.7511$. This concludes that (at a $1 \%$ significance level) the residuals does not belong to a normal distribution.

Since the hard model structure cannot describe the true system and an adequate separation using this structure cannot be achieved. By the design of this model, the hard structure ignores all frequency dependent information. It is the ignored frequency dependent part that is visible as the non-stationary variation seen in the residual sequence. This means that if this structure is used alone, all (frequency dependent) material attenuation and dispersion information is lost.

In Fig. 4, estimation results are presented for the combined model structure (30), with $P_{0}=30$. The results are presented for the case when $n_{a}=15, n_{r}=5$ and $n_{b}=3$ given by the MDL criterion, Section 3. For this model a good fit can be seen in Fig. 4a and only stationary variation is visible in the residuals in Fig. $4 \mathrm{~b}$. The residual analysis presented in Fig. $4 \mathrm{c}$ indicates a slight presence of high frequency correlation, as the residual correlation slightly violates the confidence region close to $k=0$. This violation can be eliminated if the model order is allowed to increase further, however, the MDL criterion indicates that the appropriate model order is found and the slightly observed

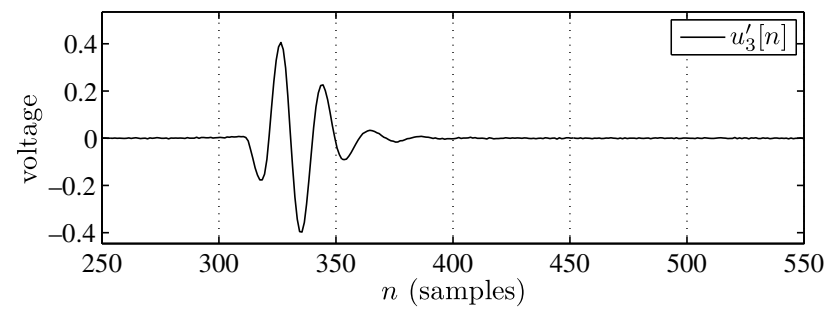

Fig. 2. The measured reference signal $u_{3}^{\prime}[n]$, defined in Section 2 as the echo from the buffer rod, see Fig. 1.
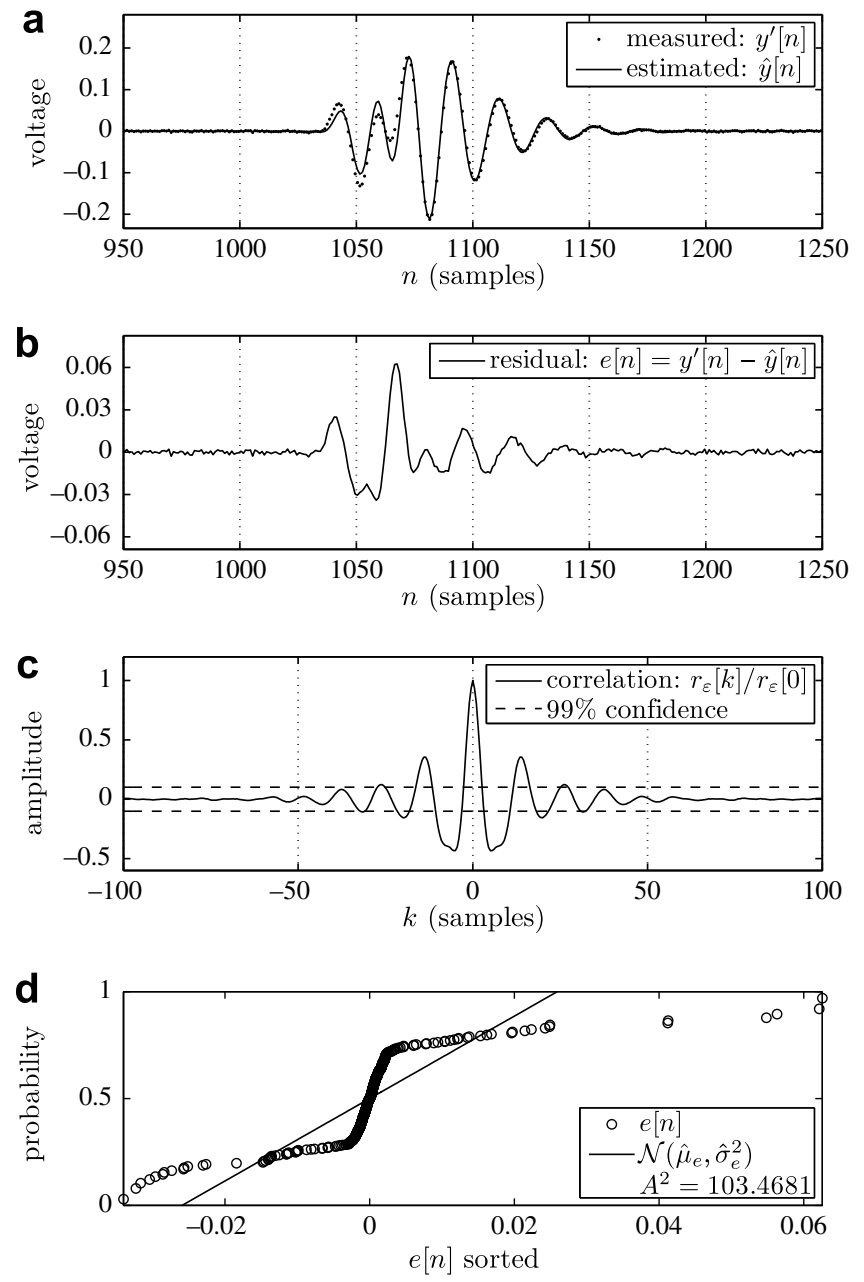

Fig. 3. Estimation results for the high viscous transmission fluid using the hard model structure in (30), with $n_{a}=n_{b}=n_{r}=1$ and $P_{0}=30$ : (a) measured response signal $y^{\prime}[n]$ versus the estimated response signal $\hat{y}[n]$ (obtained using (1) and (30)); (b) the residuals; (c) whiteness test of the weighted residuals, Appendix A.5, the dashed lines mark the $99 \%$ confidence region for $k \neq 0$; (d) normal probability plot of the residuals in (b), where $A^{2}$ is the Anderson-Darling test statistic.

correlation is at a frequency range far from the ultrasonic signals.

Fig. 4d shows a normal probability plot of the residuals. From this plot no significant deviation from the normal line can be observed, indicating a normal distribution. The Anderson-Darling test [19] for normality gives a test statistic of $A^{2}=0.1813$ which is well below the $\alpha=0.01$ threshold of $A_{\text {critical }}^{2}=0.7511$. This concludes that (at a $1 \%$ significance level) it is very unlikely that the residuals does not belong to a normal distribution.

In Fig. 5a and b, estimation results of the soft models are presented. A slight frequency dependency can be noticed in both $\widetilde{B}$ and $\widetilde{R}_{12}$, and a magnitude below one. On the other hand, a large frequency dependency can be observed in $\widetilde{A}$ and with a magnitude larger than one. This is expected since the emitted signal $\tilde{u}_{1}$ is unknown, Fig. 1, and a part of $\widetilde{A}$ in (23) has to compensate for the reference signal's propagation. The estimated dead-time for the 

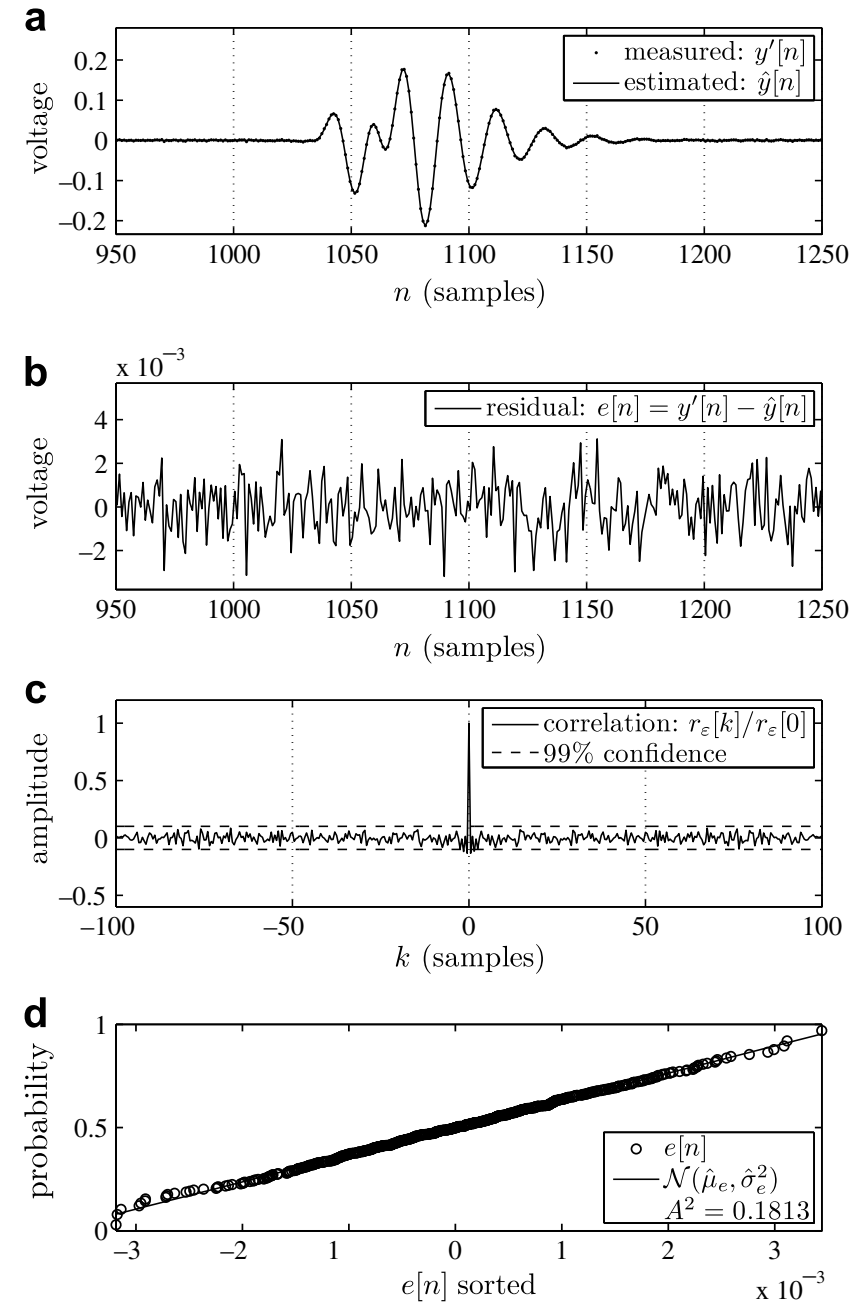

Fig. 4. Estimation results for the high viscous transmission fluid using the combined model structure in (30), with $n_{a}=15, n_{r}=5, n_{b}=3$ and $P_{0}=30$ : (a) measured response signal $y^{\prime}[n]$ versus the estimated response signal $\hat{y}[n]$ (obtained using (1) and (30)); (b) the residuals; (c) whiteness test of the weighted residuals, Appendix A.5, the dashed lines mark the $99 \%$ confidence region for $k \neq 0$; (d) normal probability plot of the residuals in (b), where $A^{2}$ is the Anderson-Darling test statistic.

transmission fluid is $\hat{\tau}_{2} / T_{\mathrm{s}}=9.65$ samples. The linearphase restriction of $\hat{\widetilde{R}}_{12}$ is visible with a constant phase of $\pi$, i.e., the frequency response is negative and real valued. In Fig. 5c, the magnitudes of the reference and response signals are shown, to give an indication of the frequency area of interest.

Once the parameter vector is estimated and the model is validated, the coinciding signals can be separated using the relationships derived in Section 4, see Fig. 6.

In Fig. 7, the sequential summation of first six separated signals, $\sum_{p=1}^{P} \hat{y}_{p}[n]$ for $P=1,2, \ldots, 6$, is compared with the measured response signal $y^{\prime}[n]$. By the definition of the model in (30), the estimated response signal $\hat{y}[n]$ is the summation of the first $P_{0}=30$ contributions estimated to be observed by the measurement window. However, as Fig. 7 reveals, only a fraction of these have significant amplitudes to contribute to the summation in building
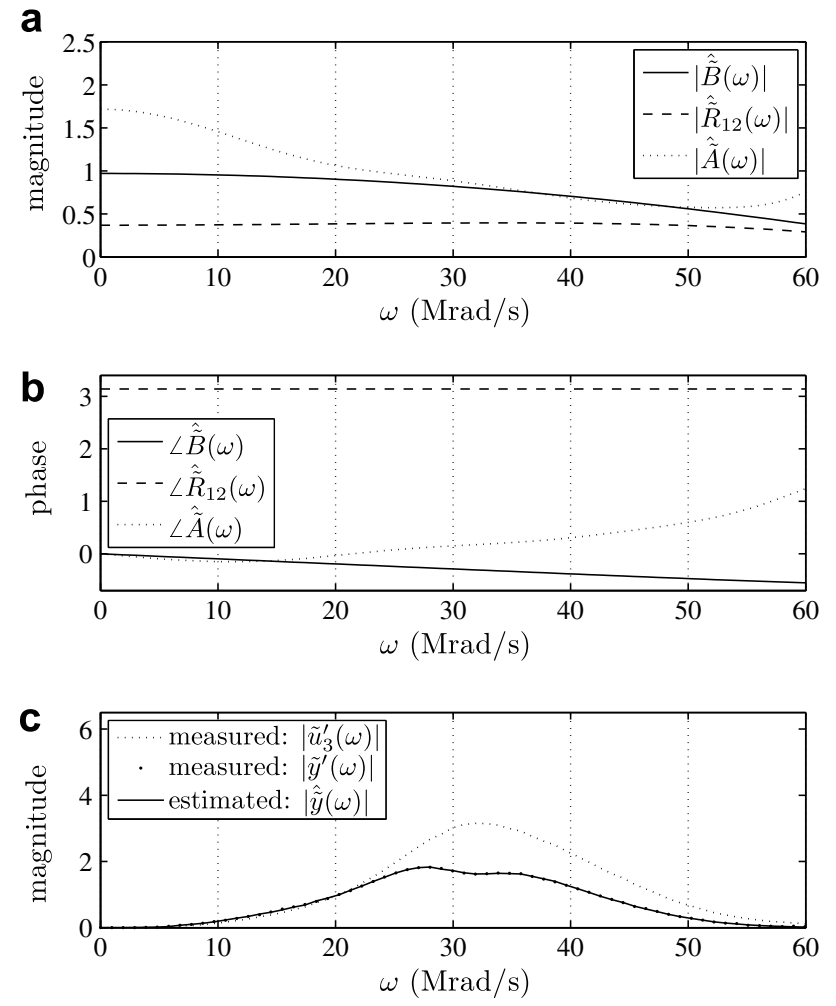

Fig. 5. Estimation results for high viscous transmission fluid: (a) magnitude response of the estimated soft models; (b) phase response of the estimated soft models; (c) magnitude of the measured and estimated signals.

the response. The rate at which the magnitudes decrease depends on the values of $\left|\widetilde{R}_{12} \widetilde{B}\right|$. For samples with lowattenuation and significantly different acoustic impedance than the surrounding media, e.g. a steel plate in water, the magnitude of the feedback term $\left|\widetilde{R}_{12} \widetilde{B}\right|$ is closer to 1 and more terms would be significant. In Section 7.5, a discussion follows on how to deal with truncated response measurements for highly reverberant samples.

Given $\hat{\boldsymbol{\theta}}$, the estimated reference and response SNR for the transmission fluid experiment are $\mathrm{SNR}_{\tilde{u}_{3}}=62.4 \mathrm{~dB}$ and $\mathrm{SNR}_{\tilde{y}}=58.2 \mathrm{~dB}$, respectively, see Appendix A.4.

\subsection{Short dispersive reverberation: separation and estimation results in glycerin}

Fig. 8 shows the measured reference signal $u_{3}^{\prime}[n]$ for the glycerin experiment, using the $5 \mathrm{MHz}$ transducer. There is a slight difference between this reference signal, and the reference signal measured in the previous experiment, Fig. 2. The main two reasons for this difference is alignment and temperature variations between the two experiments. See Section 7.1, for a detailed discussion of these variations.

Fig. 9 shows the estimation results for glycerin when the hard model structure is used to estimate the response signal. As in Fig. 3, the hard model structure cannot describe the true system and an adequate separation using this structure cannot be achieved. 


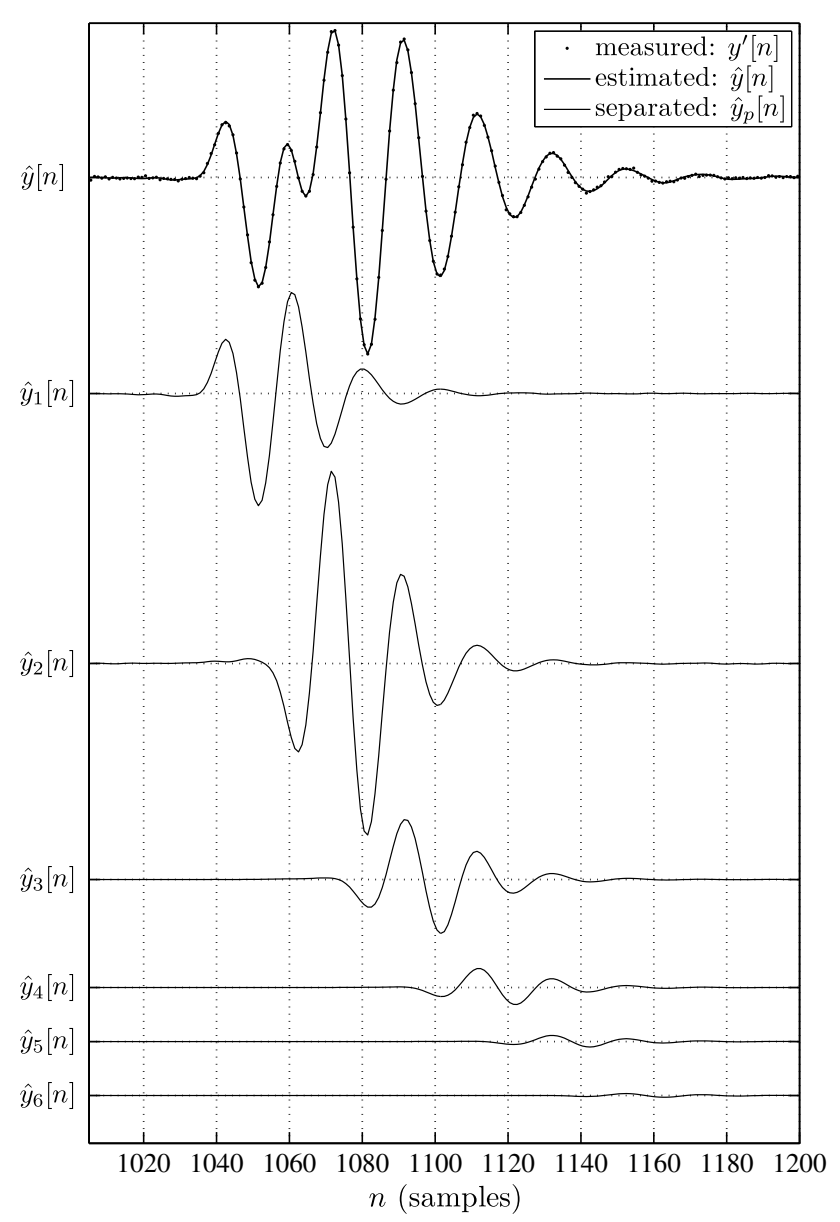

Fig. 6. Separation of the first six coinciding signals for high viscous transmission fluid, using the estimated parameters from the combined model structure, see Section 4 . The measured response signal $y^{\prime}[n]$ is plotted as dots. The bold line depicts the estimated response signal $\hat{y}[n]$ in Fig. $4 \mathrm{a}$, defined as the summation of all the $P_{0}=30$ coinciding signals fitted within the measurement window (28). The thin lines represent the separated signals $\hat{y}_{p}[n]$, using (31) together with (32) and (33).

In Fig. 10, estimation results are presented for glycerin using the combined model structure (30), with $P_{0}=30$. The results are presented for the case when $n_{a}=13$, $n_{r}=3$ and $n_{b}=3$ given by the MDL criterion. For this model a good fit can be seen in Fig. 10a and an examination and analysis of the residuals in Fig. 10b-d does not indicate any components that might originate from the ultrasonic waveforms.

In Fig. 11a and b, estimation results of the soft models are presented for glycerin. The magnitude of the reflection coefficient is small. This is why glycerin is often used as a coupling fluid, especially for polymers. The estimated dead-time for glycerin is $\hat{\tau}_{2} / T_{\mathrm{s}}=7.11$ samples.

In Fig. 12, the coinciding signals are separated. As a consequence of the matching impedances between the buffer rod and glycerin, the feedback term $\left|\widetilde{R}_{12} \widetilde{B}\right|$ is small, and only a short reverberation is visible. Most of the energy is transmitted through the sample and reflected at bottom boundary and propagated back as $\hat{y}_{2}[n]$.

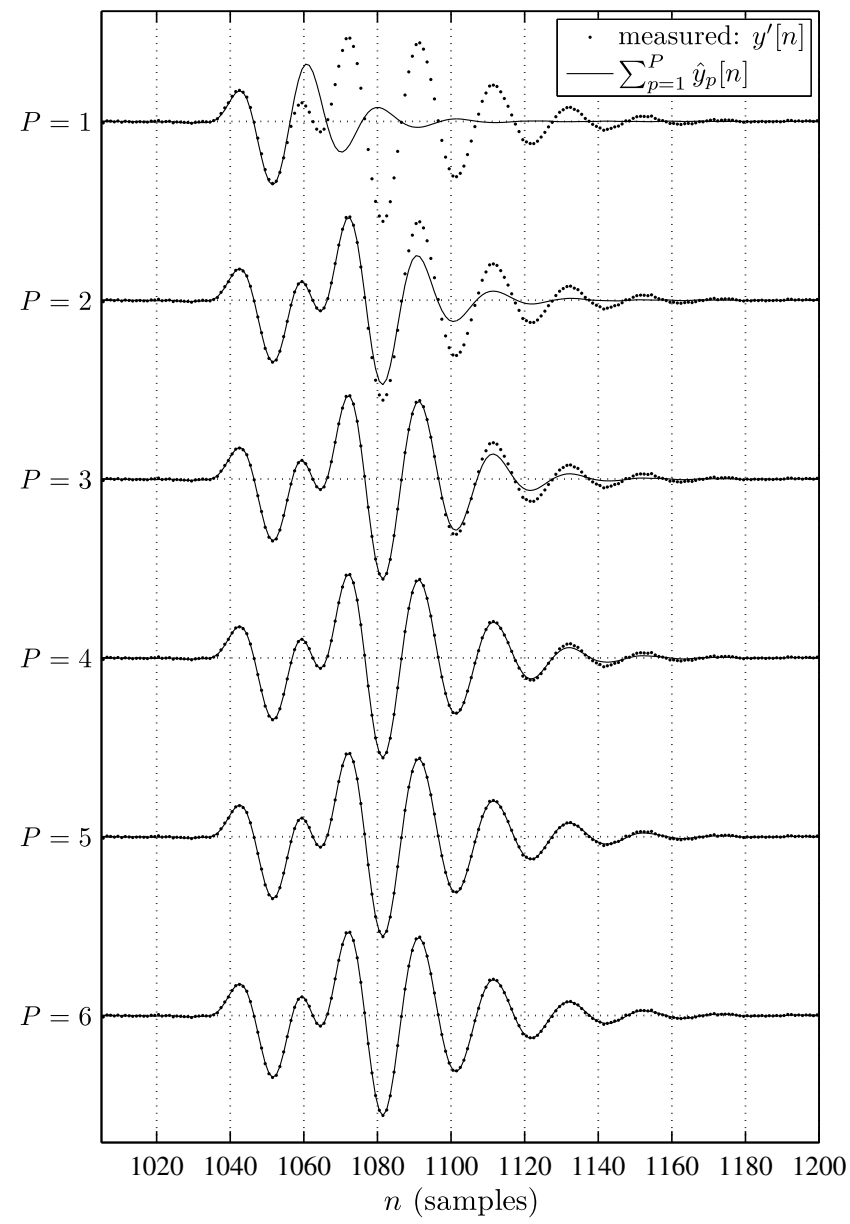

Fig. 7. Summation of the first six separated signals in Fig. 6. The measured response signal $y^{\prime}[n]$ is plotted as dots. The thin lines represent the summation of the first $P$ separated signals $\sum_{p=1}^{P} \hat{y}_{p}[n]$.

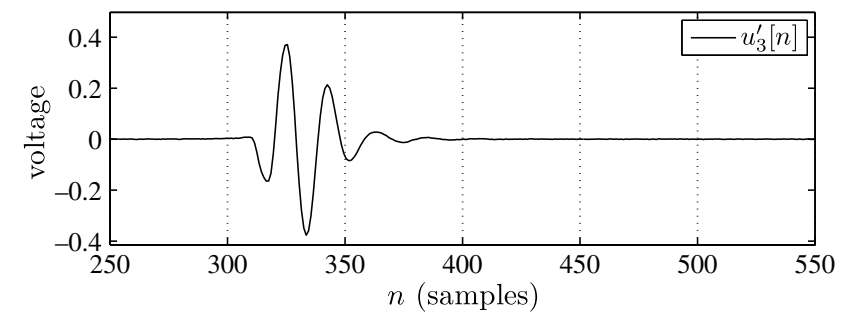

Fig. 8. The measured reference signal $u_{3}^{\prime}[n]$, defined in Section 2 as the echo from the buffer rod, see Fig. 1.

The short reverberation is also visible in Fig. 13, as only the first few contributions can describe most of the measured response.

Given $\hat{\boldsymbol{\theta}}$, the estimated reference and response SNR for the experiment in glycerin are $\mathrm{SNR}_{\tilde{u}_{3}}=65.7 \mathrm{~dB}$ and $\mathrm{SNR}_{\tilde{y}}=62.3 \mathrm{~dB}$, respectively, see Appendix A.4.

\subsection{Estimating acoustic properties}

In Figs. 14 and 15, estimates of the acoustic properties are shown for both samples, using the equations in Section 

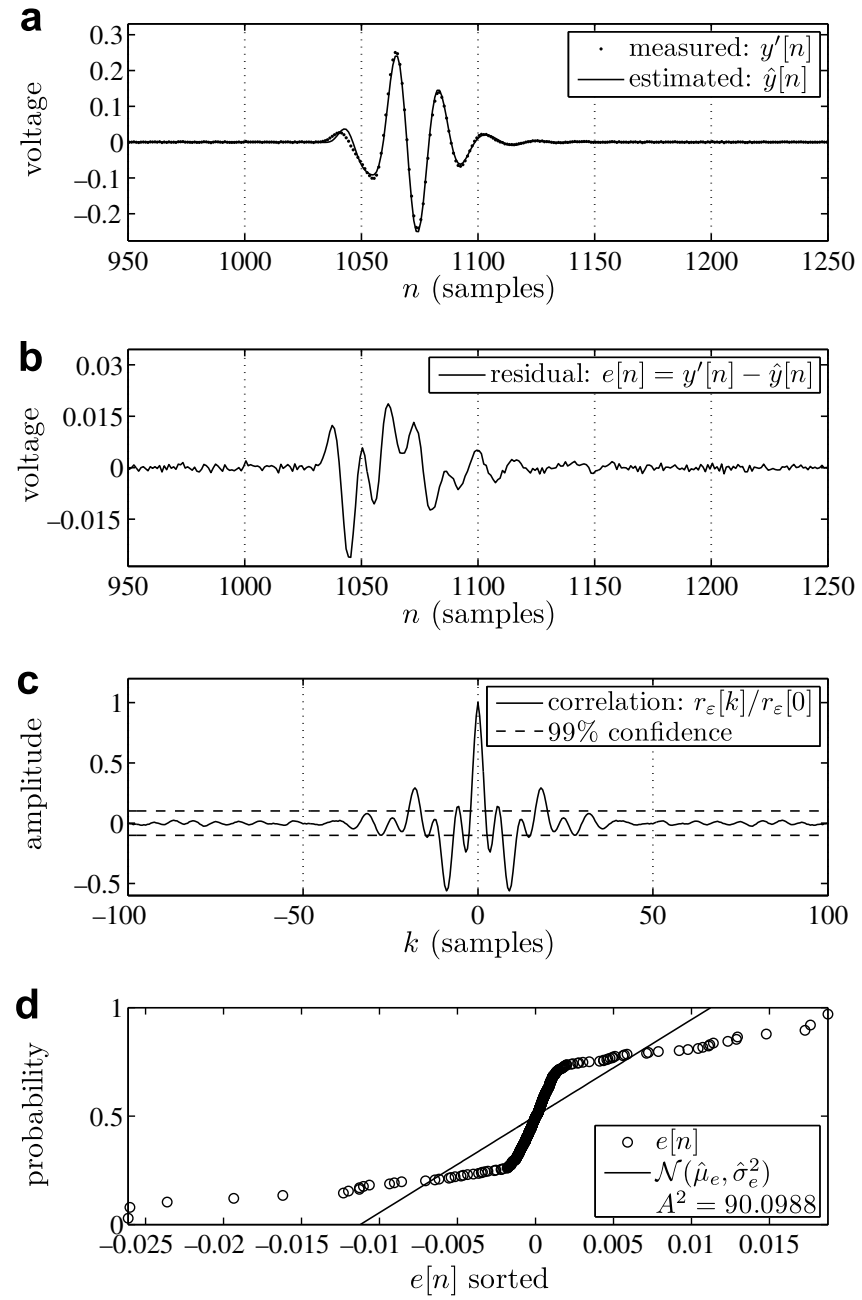

Fig. 9. Estimation results for glycerin using the hard model structure in (30), with $n_{a}=n_{r}=n_{b}=1$ and $P_{0}=30$ : (a) measured response signal $y^{\prime}[n]$ versus the estimated response signal $\hat{y}[n]$ (obtained using (1) and (30)); (b) the residuals; (c) whiteness test of the weighted residuals, Appendix A.5, the dashed lines mark the $99 \%$ confidence region for $k \neq 0$; (d) normal probability plot of the residuals in (b), where $A^{2}$ is the Anderson-Darling test statistic.

4.1, with the rough assumption that $\hat{\widetilde{R}}_{23}=1$ (steel) and $\widetilde{E}=1$ (neglecting diffraction and misalignment effects).

\section{Discussion}

This section begins with a discussion of issues concerning the estimated soft models. The discussion continues with comments on characterization of single layers within the measurement structure. The problems with colored measurement noise are also addressed. Possible model extensions and parameterizations are mentioned, followed by comments on how to deal with truncated measurements.

\subsection{Parameter estimation and sign invariance}

Examining the terms in (23), one might expect that the estimated model for $\widetilde{A}$ should be identical for both experiments, since $\widetilde{A}$ describes effects related only to the first two
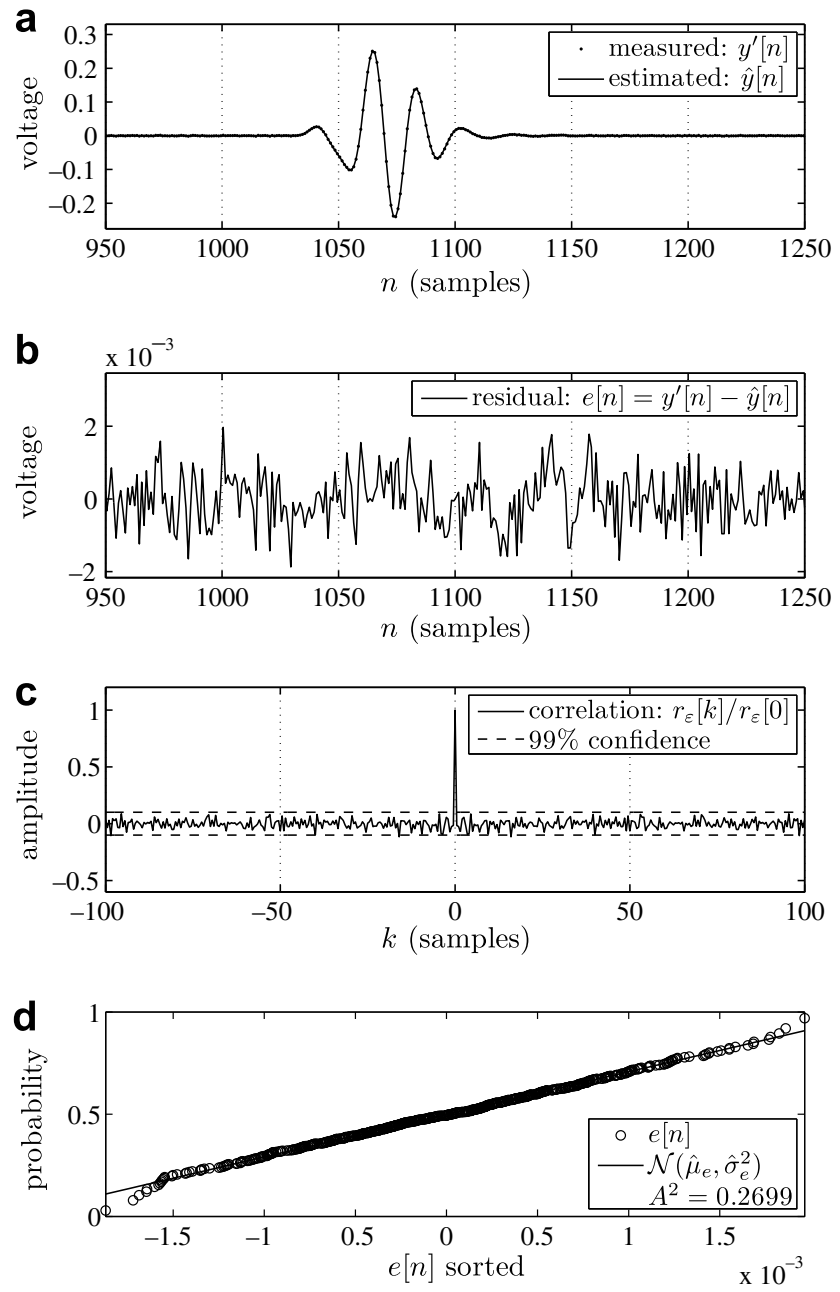

Fig. 10. Estimation results for glycerin using the combined model structure in (30), with $n_{a}=13, n_{r}=3, n_{b}=3$ and $P_{0}=30$ : (a) measured response signal $y^{\prime}[n]$ versus the estimated response signal $\hat{y}[n]$ (obtained using (1) and (30)); (b) the residuals; (c) whiteness test of the weighted residuals, Appendix A.5, the dashed lines mark the $99 \%$ confidence region for $k \neq 0$; (d) normal probability plot of the residuals in (b), where $A^{2}$ is the Anderson-Darling test statistic.

layers. However, identical $\widetilde{A}$ is obtained only if both experiments have the same alignment error, measurement temperature, and no measurement noise. Since the SNR is reasonably high within the exited frequency area, the difference in alignment and temperature is estimated to be the main cause of the slightly observed difference in the frequency response of $\widetilde{A}$.

When changing the sample fluid, the measurement cell in Fig. 1 is dismounted and mounted back again. This might produce slightly different alignment (water thickness) for the experiments. The variation in water thickness due to the mounting/dismounting step is estimated to be very small, less than $\pm 0.1 \mathrm{~mm}$. However, this variation corresponds to a phase shift of \pm 13.5 samples $( \pm 0.135 \mu \mathrm{s})$ of the measured reference signal $u_{3}^{\prime}[n]$.

The variation in the surrounding temperature is estimated to be less than $\pm 1{ }^{\circ} \mathrm{C}$, corresponding to a phase shift of \pm 7 samples $( \pm 0.07 \mu \mathrm{s})$. This phase shift is the result of a 

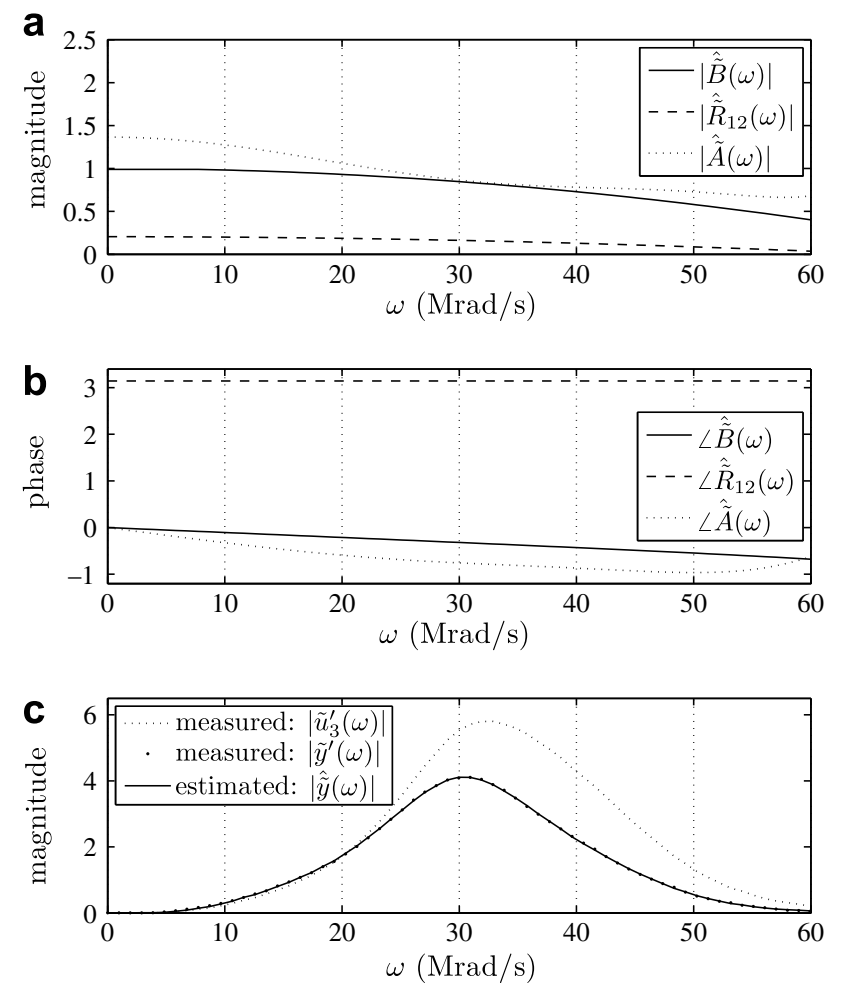

Fig. 11. Estimation results for glycerin: (a) magnitude response of the estimated soft models; (b) phase response of the estimated soft models; (c) magnitude of the measured and estimated signals.

change in the speed of sound in water [20] (a change of $1{ }^{\circ} \mathrm{C} \approx 5 \mathrm{~m} / \mathrm{s}$ ). Temperature variations also change the acoustic impedance of the PMMA buffer rod [21], resulting in a change of the reflection term $\widetilde{R}_{01}$ in $\widetilde{A}$.

The difference in alignment and temperature generates a slightly different reference signal, briefly mentioned in Section 6.2. In Fig. 16, a comparison can be seen between the measured reference signals. The observed difference is a slight change in phase $(\approx 1.5$ samples $)$ and magnitude, coherent with the phase and magnitude changes observed in $\widetilde{A}$, see Figs. 5 and 11 . It is difficult to say whether this difference is due to temperature variations or mounting/ dismounting effects (or both).

Worth mentioning is also the sign invariance for the soft models within the combined model structure in (22) or (30). Changing the sign of all the soft models $\widetilde{B}, \widetilde{R}$, and $\widetilde{A}$, does not change the frequency response of the combined model structure, nor the separation results. This sign invariance is a consequence of not knowing the true emitted signal $\tilde{u}_{1}$. However, the signs can easily be compensated for afterwards, if the sign of one soft model is known from a priori information. For example in the measurement setup used in this study, the signs of both $\widetilde{R}_{01}$ and $\widetilde{R}_{23}$ are known in advance, resulting in knowing the signs of $\widetilde{A}$ and $\widetilde{B}$ from (23) and (24).

\subsection{Characterization of layer properties}

In this paper we focus on testing the separability of the proposed technique on dispersive overlapping waveforms

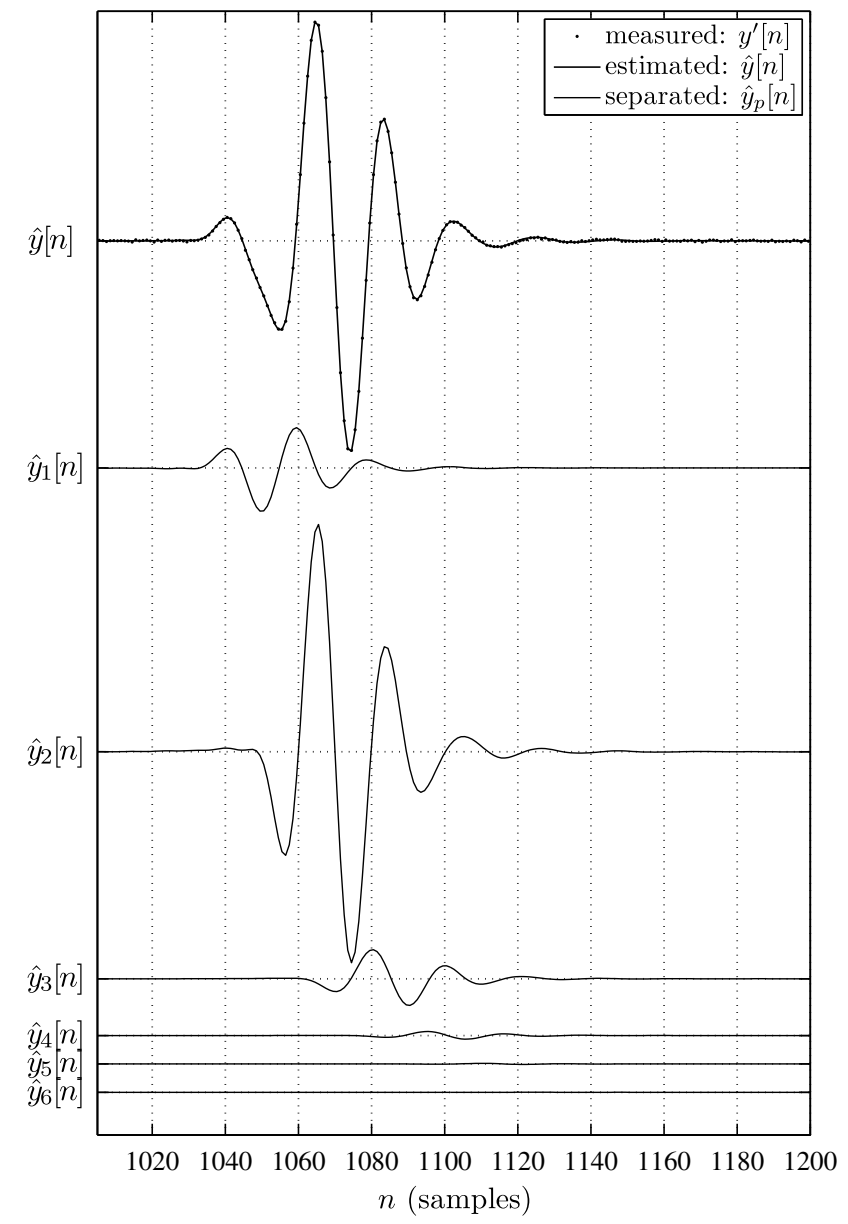

Fig. 12. Separation of the first six coinciding signals using the estimated parameters from the combined model structure, see Section 4. The measured response signal $y^{\prime}[n]$ is plotted as dots. The bold line depicts the estimated response signal $\hat{y}[n]$ in Fig. 10a, defined as the summation of all the $P_{0}=30$ coinciding signals, fitted within the measurement window. The thin lines represent the separated signals $\hat{y}_{p}[n]$, using (31) together with (32) and (33).

with different reverberation. When the objective is to characterize the material properties of the third layer only, changes in temperature and the surrounding layers (alignments and/or reflector) are undesirable. The only information of the third layer is embedded in the soft model $\widetilde{B}=\widetilde{M}_{2} \widetilde{E} \widetilde{R}_{23}$, see (24). From the analysis we can see that the diffraction and alignment part $\widetilde{E}$ in (17) depends on the total number of propagated wavelengths $\tilde{\xi}$, hence, changes in temperature and propagation distance (due to transducer alignment) will affect $\widetilde{B}$ even though $\widetilde{M}_{2}$ are unchanged. However, for thin layers and small changes we should expect only a minor change in $\widetilde{E}$ and good characterization repeatability, but for large variations $\widetilde{B}$ is affected as well. When focus is only to characterize the third layer, the measurement setup should be designed for this purpose so that the influence of $\widetilde{E}$ and $\widetilde{R}_{23}$ are very small (or known in advance from calibration).

To perform a complete randomized experiment, where all the layers (and experimental conditions) are varied to 


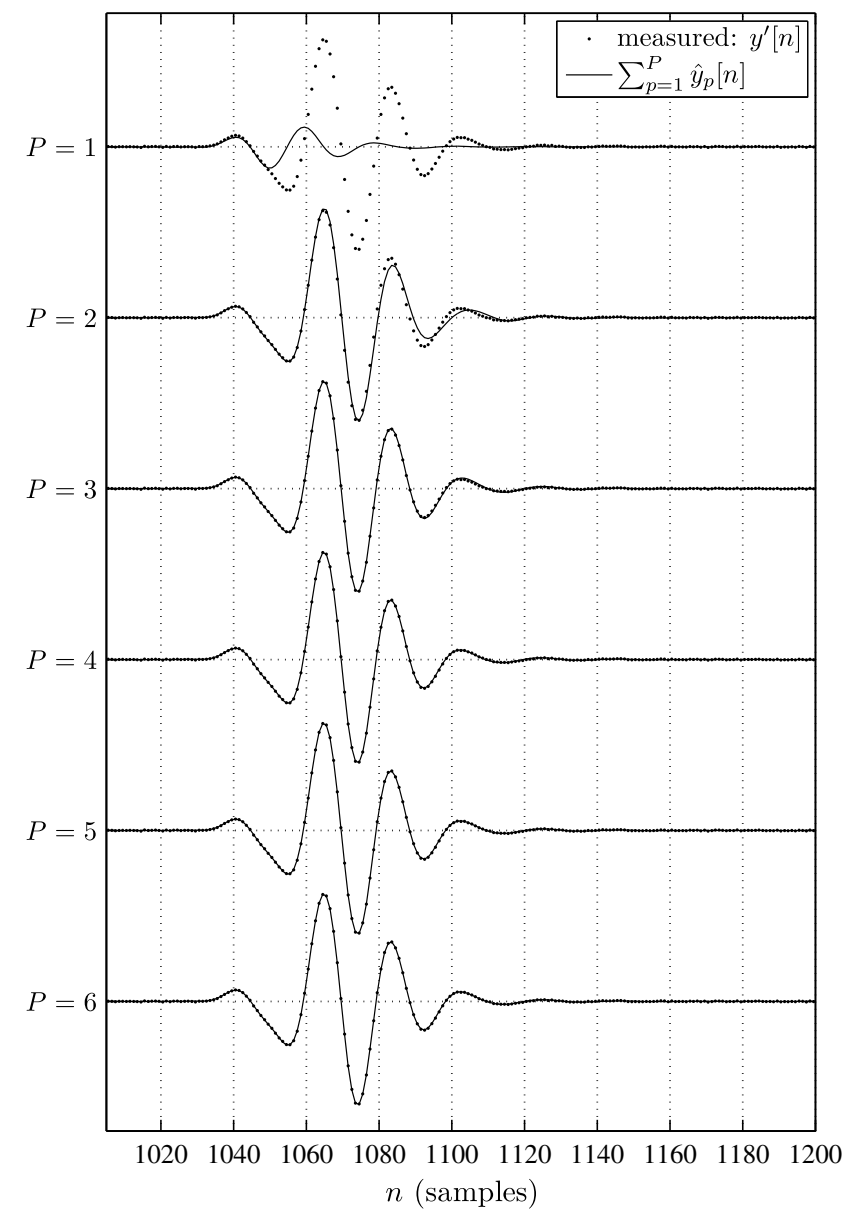

Fig. 13. Summation of the first six separated signals in Fig. 12. The measured response signal $y^{\prime}[n]$ is plotted as dots. The thin lines represent the summation of the first $P$ separated signals $\sum_{p=1}^{P} \hat{y}_{p}[n]$.

a

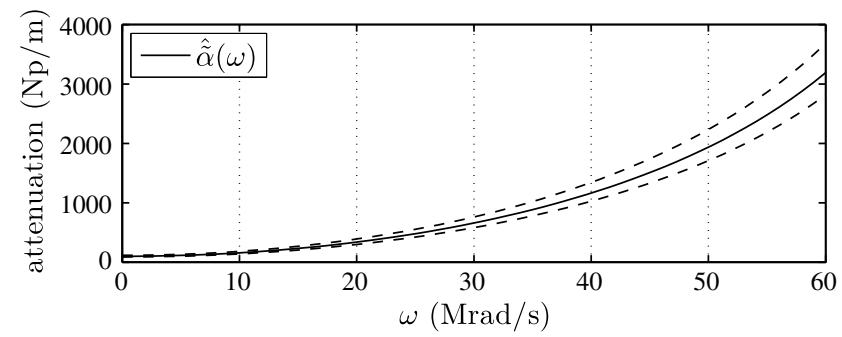

b

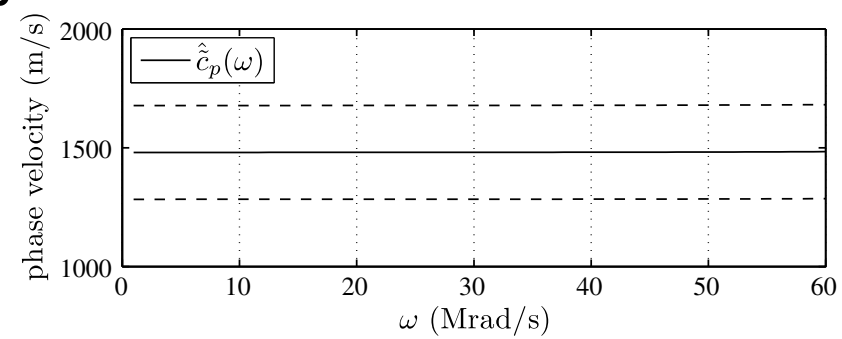

Fig. 14. Estimation results for the transmission fluid: (a) attenuation; (b) phase velocity. Dashed lines represent the area related to the thickness uncertainty $d_{2}=0.15 \pm 0.02 \mathrm{~mm}$, Section 5 . a

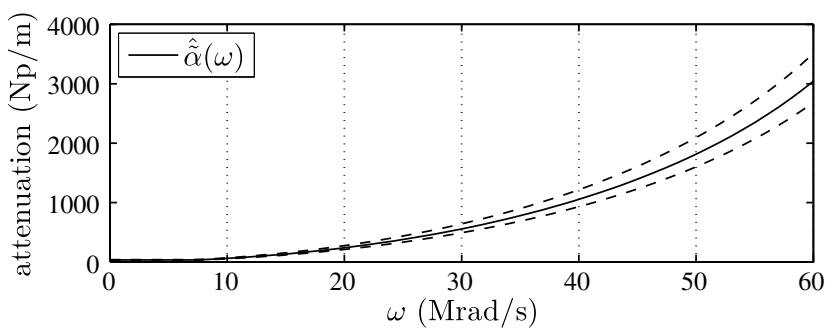

b

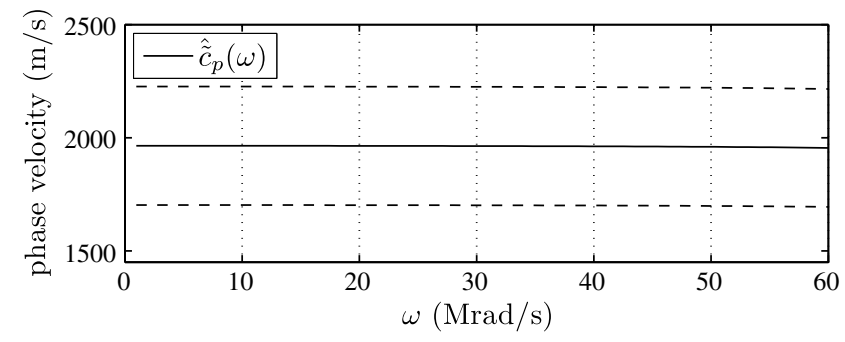

Fig. 15. Estimation results for glycerin: (a) attenuation; (b) phase velocity. Dashed lines represent the area related to the thickness uncertainty $d_{2}=0.15 \pm 0.02 \mathrm{~mm}$, Section 5 .

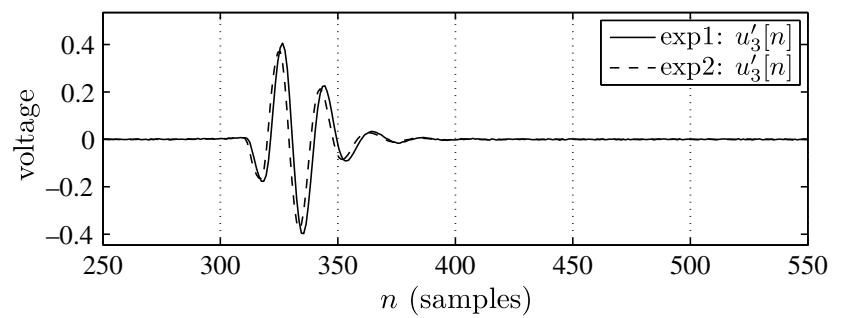

Fig. 16. Comparison of the measured reference signal in Fig. 2 and in Fig. 8.

look for independent and joint effects of the sub-elements within the soft models, is of course very interesting, but very extensive and will be addressed in future studies.

\subsection{Uncorrelated residuals}

As noticed by the visual examination of the residuals in Figs. 4, 10, colored components might be present. Even though the noticeable variation does not seem to originate from the emitted signals, it could indicate that the assumed noise characteristics of additive white Gaussian noise is too restrictive. However, one must remember that the shown residuals $e[n]$ should contain a natural correlation, since the reference noise is colored by the model $\tilde{h}(\boldsymbol{\theta}, \omega)$. Assume that the correct model is found, i.e., $\tilde{y}(\omega)=\tilde{h}(\boldsymbol{\theta}, \omega) \tilde{u}_{3}(\omega)$ then

$$
\begin{aligned}
\tilde{e}(\omega) & =\tilde{y}^{\prime}(\omega)-\tilde{h}(\boldsymbol{\theta}, \omega) \tilde{u}_{3}^{\prime}(\omega) \\
& =\left(\tilde{y}(\omega)+\tilde{w}_{\tilde{y}}(\omega)\right)-\tilde{h}(\boldsymbol{\theta}, \omega)\left(\tilde{u}_{3}(\omega)+\tilde{w}_{\tilde{u}_{3}}(\omega)\right) \\
& =\tilde{w}_{\tilde{y}}(\omega)-\tilde{h}(\boldsymbol{\theta}, \omega) \tilde{w}_{\tilde{u}_{3}}(\omega),
\end{aligned}
$$

where $\tilde{w}_{\tilde{y}}(\omega)$ and $\tilde{w}_{\tilde{u}_{3}}(\omega)$ represents the additive measurement noise on the response and the reference, respectively, see Appendix A.3. In (42), the last noise term is colored by 
the model. This is the reason why the weighted residuals $\varepsilon[n]$, in Appendix A.5, is tested for correlation.

The assumption that the weighted residuals, is uncorrelated (white) is valid when the noise assumption of additive white Gaussian noise in Appendix A.3 applies. In presence of colored additive measurement noise, a correlation appears even though a good model is used. Common sources of coloration are applied pre-filters before sampling, and additive (non-white) disturbances from the surrounding. However, if it is possible to conduct repeated experiments with the same excitation signal under stationary conditions, the cost-function in (A.20) can be used, where block matrices in $\mathbf{C}_{\tilde{w} \tilde{w}}$ are replaced with estimates obtained from the repeated experiments [12]. For the residual analysis, the weighted residuals in (A.35) is in this case replaced with $\tilde{\boldsymbol{\varepsilon}}=\hat{\mathbf{C}}_{\tilde{\mathbf{e}} \mathbf{e}}^{-1 / 2} \tilde{\mathbf{e}}$, where $\hat{\mathbf{C}}_{\tilde{\mathbf{e}} \tilde{e}}$ is constructed using (A.24).

\subsection{Extensions and parameterizations}

The theory presented in this paper is derived for the specific measurement setup shown in Fig. 1, but the ideas of combining hard and soft modeling can easily be rewritten for other measurement setups and extended to apply for multi-layered samples. The parameterization of the soft models can be changed to a more physical structure based on the physical properties of the media, if the diffraction/ alignment factor can be neglected. If a more restrictive structure is used it is important to remember the model validation step to justify a complete separation. However, as mentioned in Section 1, a necessary condition to retrieve accurate estimates of physical parameters is that all the observed dynamics can be captured by the model. An example of this can be seen when comparing the experimental results using the inadequate hard model with the results obtained from the combined model. For example, even though $\widetilde{A}$ does not model the sample layer, neglecting its dynamical properties does however significantly influence the estimate of $\widetilde{R}_{12}$ and $\widetilde{B}$. Hence, to obtain accurate estimates of physical parameters in overlapping situations, both known and unknown effects must be captured.

Instead of using a more restrictive model directly, a more suitable approach would be to first use general soft models to obtain a complete separation, and then fit physical structures to the frequency responses of the soft models. This would separate the problem into the response of each soft model, and give an indication of were significant effects have been neglected by the physical structures. An important advantage with this approach is that neglected effects from one sub-model would not influence the estimation results of other sub-models within the structure.

\subsection{Truncated response measurements}

If the measured response is truncated, i.e. the reverberant part is still present at the end of the measurement window, errors will occur in the estimation procedure if the truncation effect is ignored. The obvious way to avoid this problem is to expand the length of the measurement window so it captures the observable transients (those above the noise floor). However, in some cases this alternative is not practical, for example, in measurements of highly reverberant samples $\left(\left|\widetilde{R}_{12} \widetilde{B}\right| \approx 1\right)$ or in situations where unwanted reflections from the surrounding structure corrupts the response after a certain point.

The second alternative is to zero pad the truncated sequence at the end, with a number of zeros that is at least larger than the time support of the reference signal. Chose the number of observed reflections $P_{0}$ so that it is large enough to capture all the reflections measured by the window, and is small enough so that the modeled response dies out within the zero padded area. When estimating the model parameters, restrict the estimation process to the samples spanned by the window.

The zero padded extension will prevent the modeled response from cyclically reappear at the beginning of the sequence, a consequence of using the DFT, while still be able to chose a $P_{0}$ large enough to capture the end part of the truncated response.

\section{Conclusions}

In this paper we use a combination of hard and soft modeling to separate coinciding dispersive ultrasonic signals. A hard model structure is derived from the physical properties of the measurement setup, and contains the basic structure which enables separation. The hard structure is expanded with soft models, in the form of FIR filters, offering a general structure to cope with dispersion, attenuation, diffraction and alignment errors from the measurement setup, making a complete separation possible. Details regarding the validity and limitations of the model and the separation results are addressed. The proposed model's ability to separate coinciding signals is demonstrated for measurements on thin dispersive samples and is validated using residual analysis. The experimental results show that a complete separation is achieved, with uncorrelated and normal distributed residuals.

\section{Appendix A}

\section{A.1. Deriving the combined model structure}

The combined model structure in (22) is derived using the relationships in (1), (14), (15), (21), (18). Starting by inserting (14), (15), (21), into (18), the following expression is obtained

$$
\begin{aligned}
& \tilde{y}=\left(\widetilde{M}_{1}^{2} \widetilde{M}_{0} \widetilde{D}\left(2 \tilde{\xi}_{1}+\tilde{\xi}_{0}\right) \widetilde{R}_{12} \widetilde{T}_{10} \mathrm{e}^{-\mathrm{j} \omega\left(2 \tau_{1}+\tau_{0}\right)}\right. \\
& +\widetilde{M}_{1}^{2} \widetilde{M}_{2}^{2} \widetilde{M}_{0} \widetilde{D}\left(2 \tilde{\xi}_{1}+2 \tilde{\xi}_{2}+\tilde{\xi}_{0}\right) \\
& \left.\times \frac{\widetilde{T}_{12} \widetilde{R}_{23} \widetilde{T}_{21} \widetilde{T}_{10} \mathrm{e}^{-\mathrm{j} \omega\left(2\left(\tau_{1}+\tau_{2}\right)+\tau_{0}\right)}}{1-\widetilde{R}_{21} \widetilde{R}_{23} \widetilde{M}_{2}^{2} \widetilde{E} \mathrm{e}^{-\mathrm{j} \omega 2 \tau_{2}}}\right) \frac{\widetilde{D}\left(\tilde{\xi}_{0}\right) \widetilde{T}_{01}}{\widetilde{M}_{0} \widetilde{D}\left(2 \widetilde{\xi}_{0}\right) \widetilde{R}_{01}} \mathrm{e}^{\mathrm{j} \omega \tau_{0}} \tilde{u}_{3} .
\end{aligned}
$$


The terms $\widetilde{M}_{0}$ and $\tau_{0}$ in (A.1) are canceled. Using the relationships, $\widetilde{R}_{l m}=-\widetilde{R}_{m l}$ and $\widetilde{T}_{l m}=1+\widetilde{R}_{l m}$, the linear system in (A.1), describing the relationship in (1), can be expressed as

$$
\begin{aligned}
\tilde{h}(\boldsymbol{\theta})= & \left(\widetilde{R}_{12}+\widetilde{M}_{2}^{2} \frac{\widetilde{D}\left(2 \tilde{\xi}_{1}+2 \tilde{\xi}_{2}+\tilde{\xi}_{0}\right)}{\widetilde{D}\left(2 \tilde{\xi}_{1}+\tilde{\xi}_{0}\right)} \frac{\left(1-\widetilde{R}_{12}^{2}\right) \widetilde{R}_{23} \mathrm{e}^{-\mathrm{j} \omega 2 \tau_{2}}}{1+\widetilde{R}_{12} \widetilde{R}_{23} \widetilde{M}_{2}^{2} \widetilde{E} \mathrm{e}^{-\mathrm{j} \omega 2 \tau_{2}}}\right) \\
& \times \widetilde{M}_{1}^{2} \frac{\widetilde{D}\left(2 \tilde{\xi}_{1}+\tilde{\xi}_{0}\right) \widetilde{D}\left(\tilde{\xi}_{0}\right)}{\widetilde{D}\left(2 \tilde{\xi}_{0}\right)} \frac{\left(1-\widetilde{R}_{01}^{2}\right)}{\widetilde{R}_{01}} \mathrm{e}^{\mathrm{j} \omega 2 \tau_{1}} .
\end{aligned}
$$

Inserting (17) and (A.2) reduces to

$$
\begin{aligned}
\tilde{h}(\boldsymbol{\theta})= & \left(\widetilde{R}_{12}+\frac{\left(1-\widetilde{R}_{12}^{2}\right) \widetilde{R}_{23} \widetilde{M}_{2}^{2} \widetilde{E} \mathrm{e}^{-\mathrm{j} \omega 2 \tau_{2}}}{1+\widetilde{R}_{12} \widetilde{R}_{23} \widetilde{M}_{2}^{2} \widetilde{E} \mathrm{e}^{-\mathrm{j} \omega 2 \tau_{2}}}\right) \\
& \times \widetilde{M}_{1}^{2} \frac{\widetilde{D}\left(2 \tilde{\xi}_{1}+\widetilde{\xi}_{0}\right) \widetilde{D}\left(\tilde{\xi}_{0}\right)}{\widetilde{D}\left(2 \tilde{\xi}_{0}\right)} \frac{\left(1-\widetilde{R}_{01}^{2}\right)}{\widetilde{R}_{01}} \mathrm{e}^{\mathrm{j} \omega 2 \tau_{1}}
\end{aligned}
$$

which is the expression for the combined model structure in (22). Ignoring diffraction and misalignment effects, material dispersion and attenuation, frequency dependent reflection and transmission, Eq. (22) reduces to (13).

\section{A.2. High-pass filtering}

To remove DC components, trends and low-frequency disturbances, the measured signals have been high-pass filtered using a discrete IIR filter with transfer function

$\tilde{H}_{\mathrm{HP}}(\omega)=\frac{1+\gamma}{2} \frac{1-\mathrm{e}^{-\mathrm{j} \omega T_{\mathrm{s}}}}{1-\gamma \mathrm{e}^{-\mathrm{j} \omega T_{\mathrm{s}}}}$,

and $\gamma=0.95$. This gives a (half magnitude) cutoff angular frequency of $3 \mathrm{Mrad} / \mathrm{s}$.

\section{A.3. The maximum likelihood estimator (MLE)}

Assume that linear acoustic applies and that the description in (1) is valid. Let

$\tilde{\mathbf{z}}^{\prime}=\tilde{\mathbf{z}}+\tilde{\mathbf{w}}$,

represent the measurement vector (complex-valued), containing the discrete Fourier transformed (DFTed) sampled reference and response signal

$\tilde{\mathbf{z}}=\left[\begin{array}{c}\tilde{\mathbf{u}}_{3} \\ \tilde{\mathbf{y}}\end{array}\right]$

assumed unknown and of equal lengths ( $N$ frequency points each), and

$\tilde{\mathbf{w}}=\left[\begin{array}{c}\tilde{\mathbf{w}}_{\tilde{u}_{3}} \\ \tilde{\mathbf{w}}_{\tilde{y}}\end{array}\right]$

represent the corresponding DFTed measurement noise. The noise vector $\tilde{\mathbf{w}}$ is assumed to be zero mean complex Gaussian distributed with the covariance matrix

$$
\mathbf{C}_{\tilde{\mathbf{w}} \tilde{\mathbf{w}}}=\mathrm{E}\left\{\tilde{\mathbf{w}} \tilde{\mathbf{w}}^{H}\right\}=\left[\begin{array}{ll}
\mathbf{C}_{\tilde{\mathbf{u}}_{3} \tilde{\mathbf{u}}_{3}} & \mathbf{C}_{\tilde{\mathbf{u}}_{3} \tilde{\mathbf{y}}} \\
\mathbf{C}_{\tilde{\mathbf{y}}_{\tilde{\mathbf{u}}}} & \mathbf{C}_{\tilde{\mathbf{y}} \tilde{\mathbf{y}}}
\end{array}\right] .
$$

The corresponding vector notation of (1) can be expressed as

$\widetilde{\mathbf{G}}(\boldsymbol{\theta}) \tilde{\mathbf{z}}=\mathbf{0}$,

where

$\widetilde{\mathbf{G}}(\boldsymbol{\theta})=[-\widetilde{\mathbf{H}}(\boldsymbol{\theta}), \mathbf{I}]$,

$\widetilde{\mathbf{H}}(\boldsymbol{\theta})=\operatorname{diag}\left(\left[\tilde{h}\left(\omega_{1}, \boldsymbol{\theta}\right), \ldots, \tilde{h}\left(\omega_{N}, \boldsymbol{\theta}\right)\right]^{\mathrm{T}}\right)$,

and $\omega_{k}$ is the $k$ th corresponding angular frequency.

The log-likelihood function [17] can be expressed as

$\ln \left(p\left(\tilde{\mathbf{z}}^{\prime} ; \tilde{\mathbf{z}}, \boldsymbol{\theta}\right)\right)=-\left(\tilde{\mathbf{z}}^{\prime}-\tilde{\mathbf{z}}\right)^{H} \mathbf{C}_{\tilde{\mathbf{w}} \tilde{\mathbf{w}}}^{-1}\left(\tilde{\mathbf{z}}^{\prime}-\tilde{\mathbf{z}}\right)-A$,

where

$A=2 N \ln (\pi)+\ln \left(\operatorname{det}\left(\mathbf{C}_{\tilde{\mathbf{w}} \tilde{\mathbf{w}}}\right)\right)$.

The log-likelihood function should be maximized under the constraint given in (A.9), [12], resulting in a minimization of the Lagrangian (for complex multipliers)

$$
\begin{aligned}
L\left(\boldsymbol{\theta}, \mathbf{C}_{\tilde{\mathbf{w}} \tilde{\mathbf{w}}}, \tilde{\mathbf{z}}, \tilde{\boldsymbol{\lambda}}\right)= & \left(\tilde{\mathbf{z}}^{\prime}-\tilde{\mathbf{z}}\right)^{H} \mathbf{C}_{\tilde{\mathbf{w}} \tilde{\mathbf{w}}}^{-1}\left(\tilde{\mathbf{z}}^{\prime}-\tilde{\mathbf{z}}\right)+A \\
& +\tilde{\boldsymbol{\lambda}}^{H} \widetilde{\mathbf{G}}(\boldsymbol{\theta}) \tilde{\mathbf{z}}+\tilde{\boldsymbol{\lambda}}^{\mathrm{T}} \widetilde{\mathbf{G}}^{*}(\boldsymbol{\theta}) \tilde{\mathbf{z}}^{*} .
\end{aligned}
$$

Closed-form expressions for the estimates of $\tilde{\mathbf{z}}$ and $\tilde{\lambda}$ can be found by examining the critical points of (A.13), reducing the optimization problem to a minimization with respect to $\boldsymbol{\theta}$ and $\mathbf{C}_{\tilde{w} \tilde{w}}$ only. At the critical points the following relationship holds

$$
\begin{aligned}
& \frac{\partial L}{\partial \tilde{\mathbf{z}}^{*}}=-\mathbf{C}_{\tilde{\mathbf{w}} \tilde{\mathbf{w}}}^{-1}\left(\tilde{\mathbf{z}}^{\prime}-\tilde{\mathbf{z}}\right)+\widetilde{\mathbf{G}}^{H}(\boldsymbol{\theta}) \tilde{\boldsymbol{\lambda}}=\mathbf{0}, \\
& \frac{\partial L}{\partial \tilde{\boldsymbol{\lambda}}^{*}}=\widetilde{\mathbf{G}}(\boldsymbol{\theta}) \tilde{\mathbf{z}}=\mathbf{0} .
\end{aligned}
$$

Solving $\tilde{\lambda}$ and $\tilde{\mathbf{z}}$ in (A.14) and (A.15), the estimates are given by

$\hat{\tilde{\mathbf{z}}}=\tilde{\mathbf{z}}^{\prime}-\mathbf{C}_{\tilde{\mathbf{w}} \tilde{\mathbf{w}}} \widetilde{\mathbf{G}}^{H}(\boldsymbol{\theta}) \tilde{\boldsymbol{\lambda}}$,

$\hat{\tilde{\boldsymbol{\lambda}}}=\left(\widetilde{\mathbf{G}}(\boldsymbol{\theta}) \mathbf{C}_{\tilde{\mathbf{w}} \tilde{\mathbf{w}}} \widetilde{\mathbf{G}}^{H}(\boldsymbol{\theta})\right)^{-1} \widetilde{\mathbf{G}}(\boldsymbol{\theta}) \tilde{\mathbf{z}}^{\prime}$,

and the combination gives

$\hat{\tilde{\mathbf{z}}}=\tilde{\mathbf{z}}^{\prime}-\mathbf{C}_{\tilde{\mathbf{w}} \tilde{\mathbf{w}}} \widetilde{\mathbf{G}}^{H}(\boldsymbol{\theta})\left(\widetilde{\mathbf{G}}(\boldsymbol{\theta}) \mathbf{C}_{\tilde{\mathbf{w}} \tilde{\mathbf{w}}} \widetilde{\mathbf{G}}^{H}(\boldsymbol{\theta})\right)^{-1} \widetilde{\mathbf{G}}(\boldsymbol{\theta}) \tilde{\mathbf{z}}^{\prime}$.

Inserting (A.18) into (A.13) and using the relationship in (A.15), the cost-function reduces to a minimization of

$$
L\left(\boldsymbol{\theta}, \mathbf{C}_{\tilde{\mathbf{w}} \tilde{\mathbf{w}}}, \hat{\tilde{\mathbf{z}}}, \hat{\tilde{\boldsymbol{\lambda}}}\right)=\tilde{\mathbf{z}}^{\prime H} \widetilde{\mathbf{G}}^{H}(\boldsymbol{\theta})\left(\widetilde{\mathbf{G}}(\boldsymbol{\theta}) \mathbf{C}_{\tilde{\mathbf{w}} \tilde{\mathbf{w}}} \widetilde{\mathbf{G}}^{H}(\boldsymbol{\theta})\right)^{-1} \widetilde{\mathbf{G}}(\boldsymbol{\theta}) \tilde{\mathbf{z}}^{\prime}+A,
$$

with respect to $\boldsymbol{\theta}$ and $\mathbf{C}_{\tilde{\tilde{w}} \tilde{\mathbf{w}}}$. Evaluating the matrices in (A.19) using (A.6),(A.8),(A.10), the cost-function can be simplified to minimizing the Markov cost function [12]

$L\left(\boldsymbol{\theta}, \mathbf{C}_{\tilde{\mathbf{w}} \tilde{\mathbf{w}}}\right)=\tilde{\mathbf{e}}^{H}(\boldsymbol{\theta}) \mathbf{C}_{\tilde{\mathbf{e}} \mathbf{e}}^{-1} \tilde{\mathbf{e}}(\boldsymbol{\theta})+\ln \left(\operatorname{det}\left(\mathbf{C}_{\tilde{\mathbf{w}} \tilde{\mathbf{w}}}\right)\right)$,

where

$\tilde{\mathbf{e}}(\boldsymbol{\theta})=\tilde{\mathbf{y}}^{\prime}-\widetilde{\mathbf{H}}(\boldsymbol{\theta}) \tilde{\mathbf{u}}_{3}^{\prime}$,

$\tilde{\mathbf{y}}^{\prime}=\tilde{\mathbf{y}}+\tilde{\mathbf{w}}_{\tilde{y}}$, 
$\tilde{\mathbf{u}}_{3}^{\prime}=\tilde{\mathbf{u}}_{3}+\tilde{\mathbf{w}}_{\tilde{u}_{3}}$,

$\mathbf{C}_{\tilde{\mathbf{e}} \tilde{\mathbf{e}}}=\mathbf{C}_{\tilde{\mathbf{y}} \tilde{\mathbf{y}}}-\mathbf{C}_{\tilde{\mathbf{y}} \tilde{\mathbf{u}}_{3}} \widetilde{\mathbf{H}}^{H}(\boldsymbol{\theta})-\widetilde{\mathbf{H}}(\boldsymbol{\theta}) \mathbf{C}_{\tilde{\mathbf{u}}_{3} \tilde{\mathbf{y}}}+\widetilde{\mathbf{H}}(\boldsymbol{\theta}) \mathbf{C}_{\tilde{\mathbf{u}}_{3} \tilde{\mathbf{u}}_{3}} \widetilde{\mathbf{H}}^{H}(\boldsymbol{\theta})$.

If repeated measurements can be conducted, using the same excitation signal under stationary conditions, an estimate of the covariance matrix can easily be obtained from the repeated measurements [12] and the estimation problem reduces to a minimization of (A.20) with respect to $\boldsymbol{\theta}$, where $\mathbf{C}_{\tilde{\mathbf{w}} \tilde{\mathbf{w}}}$ is replaced by its estimate. For DFTed measurement noise the block matrices in $\mathbf{C}_{\tilde{\mathbf{w}} \tilde{\mathbf{w}}}$ (A.8) can also be approximated as diagonal $[12,22,23]$ for large data sets, resulting in a diagonal matrix $\mathbf{C}_{\tilde{\text { ẽe }}}$ in (A.24), making it easily invertible and mathematically tractable for large data sets.

However, if repeated measurements under stationary conditions cannot be performed, then estimating the full (unconstrained) covariance matrix from one measurement is difficult. If the estimation problem is restricted to the class of white Gaussian noise, assuming that $\tilde{\mathbf{w}}_{\tilde{y}}$ and $\tilde{\mathbf{w}}_{\tilde{u}_{3}}$ are independent and has the same variance $\sigma_{\tilde{w}}^{2}$ see Fig. 1, then the covariance matrix reduces to

$\mathbf{C}_{\tilde{\mathbf{w}} \tilde{\mathbf{w}}}=\sigma_{\tilde{w}}^{2}\left[\begin{array}{ll}\mathbf{I} & \mathbf{0} \\ \mathbf{0} & \mathbf{I}\end{array}\right]$,

and the optimization problem reduces to a minimization of

$L\left(\boldsymbol{\theta}, \sigma_{\tilde{w}}^{2}\right)=\tilde{\mathbf{e}}^{H}(\boldsymbol{\theta}) \mathbf{C}_{\tilde{\mathbf{e}} \tilde{\mathbf{e}}}^{-1} \tilde{\mathbf{e}}(\boldsymbol{\theta})+2 N \ln \left(\sigma_{\tilde{w}}^{2}\right)$,

where

$\tilde{\mathbf{e}}(\boldsymbol{\theta})=\tilde{\mathbf{y}}^{\prime}-\widetilde{\mathbf{H}}(\boldsymbol{\theta}) \tilde{\mathbf{u}}_{3}^{\prime}$,

$\mathbf{C}_{\tilde{\mathbf{e}} \tilde{\mathbf{e}}}=\sigma_{\tilde{w}}^{2}\left(\mathbf{I}+\widetilde{\mathbf{H}}(\boldsymbol{\theta}) \widetilde{\mathbf{H}}^{H}(\boldsymbol{\theta})\right)$.

Setting the partial derivative of (A.26) with respect to $\sigma_{\tilde{w}}^{2}$ to zero, the minimum is given by

$\hat{\sigma}_{\tilde{w}}^{2}(\boldsymbol{\theta})=\frac{1}{2 N} \tilde{\mathbf{e}}^{H}(\boldsymbol{\theta})\left(\mathbf{I}+\widetilde{\mathbf{H}}(\boldsymbol{\theta}) \widetilde{\mathbf{H}}^{H}(\boldsymbol{\theta})\right)^{-1} \tilde{\mathbf{e}}(\boldsymbol{\theta})$,

assuming that $\sigma_{\tilde{w}}^{2} \neq 0$. Inserting this closed-form expression into (A.26), the dimensionality of the minimization problem reduces to minimization of

$L\left(\boldsymbol{\theta}, \hat{\sigma}_{\tilde{w}}^{2}(\boldsymbol{\theta})\right)=2 N+2 N \ln \left(\hat{\sigma}_{\tilde{w}}^{2}(\boldsymbol{\theta})\right)$

with respect to $\boldsymbol{\theta}$. The constant, scaling factor and logarithmic operation in (A.30) can be ignored, without changing the location of the minimum point, and the optimization problem can be rewritten to minimize a weighted nonlinear least-square problem

$L(\boldsymbol{\theta})=\tilde{\mathbf{e}}^{H}(\boldsymbol{\theta})\left(\mathbf{I}+\widetilde{\mathbf{H}}(\boldsymbol{\theta}) \widetilde{\mathbf{H}}^{H}(\boldsymbol{\theta})\right)^{-1} \tilde{\mathbf{e}}(\boldsymbol{\theta})$,

with respect to $\boldsymbol{\theta}$.

\section{A.4. Signal-to-noise ratio (SNR)}

Since the measured signals have (close to) finite time support, the calculation of the signal-to-noise ratio (SNR) in this paper involves the energy of the signal (ignoring $T_{\mathrm{s}}$ ), instead of the power (or variance). The reference and response signal-to-noise ratio (SNR) are estimated as

$\mathrm{SNR}_{\tilde{u}_{3}}=\frac{\sum_{k=1}^{N}\left|\tilde{u}_{3}^{\prime}[k]\right|^{2}}{\hat{\sigma}_{\tilde{w}}^{2}}-N$,

$\mathrm{SNR}_{\tilde{y}}=\frac{\sum_{k=1}^{N}\left|\tilde{y}^{\prime}[k]\right|^{2}}{\hat{\sigma}_{\tilde{w}}^{2}}-N$,

respectively. The $-N$ term compensates for the noise contribution in the measurements $\tilde{u}_{3}^{\prime}[k]$ and $\tilde{y}^{\prime}[k]$, see (A.23) and (A.22). And $\hat{\sigma}_{\tilde{w}}^{2}$ is given by (A.29).

\section{A.5. Residual analysis}

The cost-function in (A.31) can be rewritten as

$L(\boldsymbol{\theta})=\tilde{\boldsymbol{\varepsilon}}^{H}(\boldsymbol{\theta}) \tilde{\boldsymbol{\varepsilon}}(\boldsymbol{\theta})$,

where

$\tilde{\boldsymbol{\varepsilon}}(\boldsymbol{\theta})=\left(\mathbf{I}+\widetilde{\mathbf{H}}(\boldsymbol{\theta}) \widetilde{\mathbf{H}}^{H}(\boldsymbol{\theta})\right)^{-1 / 2} \tilde{\mathbf{e}}(\boldsymbol{\theta})$

is the weighted residuals.

Because the reference signal $\tilde{\mathbf{u}}_{3}$ is corrupted with measurement noise, the residual vector $\tilde{\mathbf{e}}(\boldsymbol{\theta})=\tilde{\mathbf{y}}^{\prime}-\widetilde{\mathbf{H}}(\boldsymbol{\theta}) \tilde{\mathbf{u}}_{3}^{\prime}$ is not white, see Section 7.3. For this reason the residual analysis is performed on the weighted residuals $\tilde{\boldsymbol{\varepsilon}}(\boldsymbol{\theta})$ in (A.35), which compensates for the reference noise and the applied correlation by the model.

The residual analysis is based on the assumption that $\varepsilon[n]$ is zero mean white noise. If this is the case then the normalized test quantity

$\frac{\hat{r}_{\varepsilon}[k]}{\hat{r}_{\varepsilon}[0]} \underset{\text { asym }}{\rightarrow} \mathscr{N}(0, N)$ for $k \neq 0$

is asymptotically Gaussian distributed as $N$ grows large [24], where the estimated autocorrelation sequence (biased) is given as

$\hat{r}_{\varepsilon}[k]=\frac{1}{N} \sum_{n=1+\max (-k, 0)}^{N-\max (k, 0)} \varepsilon^{*}[n] \varepsilon[n+k]$.

Based on these assumptions, a confidence region can be plotted for the tails $(k \neq 0)$ of the normalized test quantity in (A.36).

\section{References}

[1] M. Feder, E. Weinstein, Parameter estimation of superimposed signals using the em algorithm, IEEE Trans. Acoust. Speech Signal Process. 36 (4) (1988) 477-489.

[2] R. Demirli, J. Saniie, Model-based estimation of ultrasonic echoes. Part i: Analysis and algorithms, IEEE Trans. Ultrason. Ferroelect. Freq. Contr. 48 (3) (2001) 787-802.

[3] R.J. Freemantle, R.E. Challis, J.D.H. White, A z-transform technique for thin-layer reverberation cancellation applied to ultrasonic NDT of adhered structures, Adv. Tech. Collection Interpret. NDT Data (1994) 102.

[4] L. Wang, B. Xie, S.I. Rokhlin, Determination of embedded layer properties using adaptive time-frequency domain analysis, J. Acoust. Soc. Am. 111 (6) (2002) 2644-2653. 
[5] C. Martin, J.J. Meister, M. Arditi, P.A. Farine, A novel homomorphic processing of ultrasonic echoes for layer thickness measurement, IEEE Trans. Ultrason. Ferroelect. Freq. Contr. 40 (7) (1992) 1819 1825.

[6] V.K. Kinra, V.R. Iyer, Ultrasonic measurement of the thickness, phase velocity, density or attenuation of a thin-viscoelastic plate. Part I: the forward problem, Ultrasonics 33 (1995) 95-109.

[7] V.K. Kinra, V.R. Iyer, Ultrasonic measurement of the thickness, phase velocity, density or attenuation of a thin-viscoelastic plate. Part II: the inverse problem, Ultrasonics 33 (1995) 111-112.

[8] J. Martinsson, F. Hägglund, J.E. Carlson, Separation of dispersive coinciding signals by combining hard and soft modeling, in: Proceedings of International Congress on Ultrasonics, Vienna, Austria, pp. 1563-R17, in press, doi:10.3728/ICUltrasonics.2007. Vienna.1563_martinsson.

[9] B. Porat, A Course In Digital Signal Processing, John Wiley and Sons, 1997.

[10] K. Kendall, D. Tabor, An ultrasonic study of the area of contact between stationary and sliding surfaces, in: Proceedings of the Royal Society, Series A, 1971, pp. 321-340.

[11] B.W. Drinkwater, R.S. Dwyer-Joyce, P. Cawley, A study of the interaction between ultrasound and a partially contacting solid-solid interface, in: Proceedings of the Royal Society, Series A, 1996, pp. $2613-2628$.

[12] R. Pintelton, J. Schoukens, System Identification: A Frequency Domain Approach, IEEE Press, 2001.

[13] R. Fletcher, Practical Methods of Optimization, second ed., Wiley, New York, 1991.
[14] J. Rissanen, Modelling by the shortest data description, Automatica 14 (1978) 465-471.

[15] L. Ljung, System Identification: Theory for the User, 2nd ed., Prentice-Hall, Englewood Cliffs, 1987.

[16] J. Martinsson, J.E. Carlson, J. Niemi, Model-based phase velocity and attenuation estimation in wideband ultrasonic measurement systems, IEEE Trans. Ultrason. Ferroelect. Freq. Contr. 54 (1) (2007) $138-146$.

[17] S.M. KayFundamentals of Statistical Signal Processing: Estimation Theory, vol. 1, Prentice Hall, 1993.

[18] Castrol Marine Gear Oil (SAE90, API GL5, Mercury 92-70095, OMC 1632), manufactured by Castrol in the EU. <http:// www.castrol.com>.

[19] T.W. Anderson, D.A. Darling, Asymptotic theory of certain goodness-of-fit criteria based on stochastic processes, Ann. Math. Statist. 23 (1952) 193-212.

[20] J. Lubbers, R. Graaff, A simple and accurate formula for the sound velocity in water, Ultrasound Med. Biol. 24 (7) (1998) 1065-1068.

[21] J.E. Carlson, J. van Deventer, C. Carlander, Frequency and temperature dependence of acoustic properties of polymers used in pulseecho systems, in: Proceedings of IEEE International Ultrasonics Symposium, 2003, pp. 885-888.

[22] D.R. Brillinger, Time Series: Data Analysis and TheoryHolden-Day Series in Time Series Analysis, Holden-Day, San Francisco, 1981.

[23] L. Ljung, On the estimation of transfer functions, Automatica 21 (6) (1985) 677-696.

[24] T. Söderström, P. Stoica, System Identification, Prentice-Hall, Englewood Cliffs, 1989. 